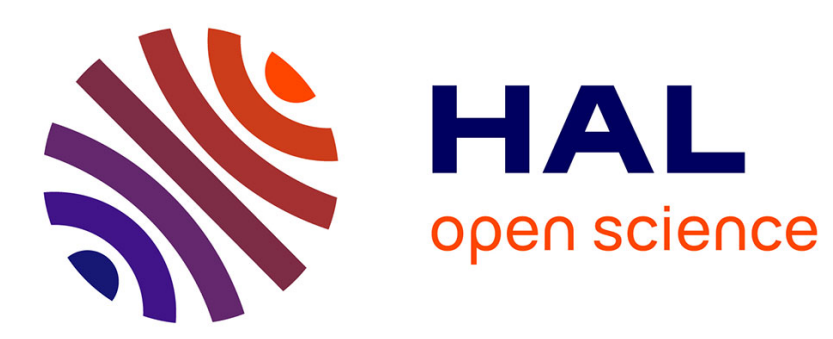

\title{
Bloch-wave homogenization for spectral asymptotic analysis of the periodic Maxwell operator
}

Kirill Cherednichenko, Sébastien Guenneau

\section{To cite this version:}

Kirill Cherednichenko, Sébastien Guenneau. Bloch-wave homogenization for spectral asymptotic analysis of the periodic Maxwell operator. Journal Waves Random Complex Media, 2007, 17 (4), pp.627651. 10.1080/17455030701551930 . hal-00186688

\section{HAL Id: hal-00186688 \\ https://hal.science/hal-00186688}

Submitted on 3 Dec 2007

HAL is a multi-disciplinary open access archive for the deposit and dissemination of scientific research documents, whether they are published or not. The documents may come from teaching and research institutions in France or abroad, or from public or private research centers.
L'archive ouverte pluridisciplinaire HAL, est destinée au dépôt et à la diffusion de documents scientifiques de niveau recherche, publiés ou non, émanant des établissements d'enseignement et de recherche français ou étrangers, des laboratoires publics ou privés. 


\title{
Bloch wave homogenisation for spectral asymptotic analysis of the periodic Maxwell operator
}

\author{
K. D. Cherednichenko*†, S. Guenneau ${ }^{\ddagger}$
}

December 3, 2007

\begin{abstract}
This paper is devoted to the asymptotic behaviour of the spectrum of the three-dimensional Maxwell operator in a bounded periodic heterogeneous dielectric medium $\mathbf{T}=[-T, T]^{3}, T>0$, as the structure period $\eta$, such that $\eta^{-1} T$ is a positive integer, tends to 0 . The domain $\mathbf{T}$ is extended periodically to the whole of $\mathbb{R}^{3}$, so that the original operator is understood as acting in a space of $\mathbf{T}$-periodic functions. We use the so-called Bloch wave homogenisation technique which, unlike the classical homogenisation method, is capable of characterising a renormalised limit of the spectrum (called the Bloch spectrum). The related procedure is concerned with sequences of eigenvalues $\Lambda_{\eta}$ of the order of the square of the medium period, which correspond to the oscillations of high-frequencies of order $\eta^{-1}$. The Bloch-wave description is obtained via the notion of two-scale convergence for bounded self-adjoint operators, and a proof of the "completeness" of the limiting spectrum is provided.
\end{abstract}

Keywords: Electromagnetic waves, periodic structures, two-scale convergence, spectrum.

\section{Introduction}

The problem of determining the effective properties of a composite periodic medium in the context of electrostatics is an old one, and has been studied extensively, starting with the paper [11] by Lorenz. However, when the electric fields exhibit time-dependence one moves into the area of electromagnetism, which is a less-developed subject, at least in the context of the effective response of periodic composite media. In this respect it may be helpful to recall that although practitioners often make use of the traditional static formulae in the dynamic regime, this technique, generally known as the "quasi-static limit", tacitly relies on a set of assumptions about the fields in the material; namely, that their wavelengths are long in comparison with the typical size of heterogeneity. In this respect it seems to be of interest to investigate the material response when the wavelengths and the average period of variations in the conducting properties become comparable, thus inducing significant scale interactions. Whether the material then supports the imposed oscillations, such as an externally applied electric signal, becomes a non-trivial question, requiring a new averaging procedure of some sort.

There are several recent examples of similar kind, where the limiting procedure has exhibited a strong dependence on the precise way in which the underlying length-scales become small, see e.g. [13], [17]. Most of them are due to some sort of contrast in the properties at different points of the composite, which makes it possible to have comparatively short waves in selected parts of the medium. It seems equally plausible to imagine, however, that the external frequency is high enough already to warrant the existence of scale interaction effects in the medium. Several works investigating this possibility have been written by Allaire and Conca, in the context of fluid-solid structures [2], [4], as well as for a scalar elliptic equation with Dirichlet boundary conditions [3]. The present paper deals with an analogous problem in the context of electromagnetic waves. In order to avoid technical complications unrelated to the aim of the present study, we restrict ourselves to the case of periodic boundary conditions, thus excluding a possibility of the emergence of a "boundary layer spectrum", see e.g. [3]. Throughout the paper we use bold type to denote various spaces of vector functions, such as $\mathbf{L}^{2}$, or $\mathbf{C}_{\text {per }}^{\infty}$; the subscript "per" will refer to the respective spaces of periodic functions. Given an operator $\mathcal{A}$, we use the notation $\sigma(\mathcal{A})$ for its spectrum.

Consider an isotropic dielectric medium occupying the domain $\mathbf{T}=[-T, T]^{3} \subset \mathbb{R}^{3}$ of fixed period $2 T>0$, which is extended periodically to $\mathbb{R}^{3}$ and composed of $\left(\eta^{-1} 2 T\right)^{3}$ periodicity cells with a small period $\eta \in \Xi$,

\footnotetext{
*School of Mathematics, Cardiff University, Senghennydd Road, Cardiff, CF24 4AG; E-mail: cherednichenkokd@cardiff.ac.uk

${ }^{\dagger}$ Corresponding author

$¥$ Department of Mathematical Sciences, University of Liverpool, Peach Street, Liverpool, L69 3BX; E-mail: guenneau@liverpool.ac.uk
} 
where the set $\Xi$ is defined by $\Xi:=\left\{\eta>0: \eta^{-1} T \in \mathbb{N}\right\}$. Such a composite medium can be a model of what is known as the photonic crystal [16]. The analysis of electromagnetic modes in such a medium amounts to looking for pairs ${ }^{1}\left(\Lambda_{\eta}, \boldsymbol{H}_{\eta}\right) \in \mathbb{R}^{+} \times \mathcal{H}_{\text {per }}(\operatorname{curl}, \mathbf{T}), \boldsymbol{H}_{\eta} \not \equiv \mathbf{0}$, such that

$$
\operatorname{curl}\left(\varepsilon^{-1}(\boldsymbol{x}, \boldsymbol{x} / \eta) \operatorname{curl} \boldsymbol{H}_{\eta}\right)=\Lambda_{\eta}^{-1} \boldsymbol{H}_{\eta},
$$

where $\varepsilon(\boldsymbol{x}, \boldsymbol{x} / \eta)$ is the (matrix) relative permittivity of the medium at the point $\boldsymbol{x}$. The set of all first elements $\Lambda_{\eta}$ of such pairs is referred to as the spectrum of (1.1), and we denote it by $\sigma_{\eta}$. We assume that $\varepsilon(\boldsymbol{x}, \boldsymbol{y}) \in C_{\mathrm{per}}\left(\mathbf{T},\left[L^{\infty}(Y)\right]^{9}\right)$, where $Y:=[0,1]^{3}$, and that $\varepsilon(\boldsymbol{x}, \boldsymbol{y}) \geq I$ for a.e. $(\boldsymbol{x}, \boldsymbol{y}) \in \mathbf{T} \times Y$, which, in the case when the medium is isotropic, means that $\varepsilon(\boldsymbol{x}, \boldsymbol{y})$ is bounded below at almost every point by the permittivity of vacuum. It follows from the above two conditions that the operator in the left-hand side of (1.1) is uniformly elliptic (or "coercive"). The equation (1.1) is understood in the weak sense, i.e.

$$
\int_{\mathbf{T}} \varepsilon^{-1}(\boldsymbol{x}, \boldsymbol{x} / \eta) \operatorname{curl} \boldsymbol{H}_{\eta}(\boldsymbol{x}) \cdot \operatorname{curl} \boldsymbol{\varphi}(\boldsymbol{x}) \mathrm{d} \boldsymbol{x}=\Lambda_{\eta}^{-1} \int_{\mathbf{T}} \boldsymbol{H}_{\eta}(\boldsymbol{x}) \cdot \boldsymbol{\varphi}(\boldsymbol{x}) \mathrm{d} \boldsymbol{x}
$$

for any $\boldsymbol{\varphi} \in \mathbf{C}_{\mathrm{per}}^{\infty}(\mathbf{T})$. Note in particular that for any solution of (1.1) one has $\operatorname{div} \boldsymbol{H}_{\eta}=0$.

In order to study the spectrum of (1.1) for each $\eta \in \Xi$, consider the Green operator $\mathcal{G}_{\eta}$ in $\mathbf{L}^{2}(\mathbf{T})$ defined for every $\boldsymbol{f} \in \mathbf{L}^{2}(\mathbf{T})$ by $\mathcal{G}_{\eta} \boldsymbol{f}=\boldsymbol{u}_{\eta}$, where $\boldsymbol{u}_{\eta}$ is the unique solution (by the Lax-Milgram lemma, see e.g. [10]) in $\mathcal{H}_{\text {per }}(\mathbf{c u r l}, \mathbf{T})$ of the problem

$$
\operatorname{curl}\left(\varepsilon^{-1}(\boldsymbol{x}, \boldsymbol{x} / \eta) \operatorname{curl} \boldsymbol{u}_{\eta}\right)+\boldsymbol{u}_{\eta}=\boldsymbol{f} .
$$

Clearly, $\mathcal{G}_{\eta}$ is self-adjoint and it can also be shown that it is compact, we can thus write $\sigma\left(\mathcal{G}_{\eta}\right)=\{0,1\} \bigcup\left\{\tilde{\Lambda}_{\eta}^{k}\right\}_{k \geq 1}$, where for each $\eta$ the sequence $\tilde{\Lambda}_{\eta}^{k}$ converges to 0 as $k \rightarrow \infty$. In the above union we include every eigenvalue as many times as is its multiplicity, so that to each $\tilde{\Lambda}_{\eta}^{k}$ is associated a normalised eigenfunction ${ }^{2} \boldsymbol{u}_{\eta}^{k} \in \mathbf{L}_{\text {sol }}^{2}(\mathbf{T})$ such that $\left\|\boldsymbol{u}_{\eta}^{k}\right\|_{\mathbf{L}^{2}(\mathbf{T})}=1$ and the family $\left\{\boldsymbol{u}_{\eta}^{k}\right\}_{k \geq 1}$ is an orthonormal basis of $\mathbf{L}_{\text {sol }}^{2}(\mathbf{T})$. The eigenvalues $\tilde{\Lambda}_{\eta}^{k}$ are related to the eigenvalues of (1.1) by the formula $\tilde{\Lambda}_{\eta}^{k}=\left(\Lambda_{\eta}^{k}+1\right)^{-1} \Lambda_{\eta}^{k}$.

The aim of this paper is to study the asymptotic behaviour of the spectrum $\sigma_{\eta}$ when the period $\eta$ goes to zero along the set $\Xi$. In what follows we focus on high frequencies, but it seems worth giving an outline of the main result for the "low-frequency" limit. In this case it is known (see e.g. [9], [8]) that when $\boldsymbol{f} \in \mathbf{L}^{2}(\mathbf{T})$, the solutions $\boldsymbol{u}_{\eta}$ of (1.2) converge strongly in $\mathbf{L}^{2}(\mathbf{T})$ to a limit $\boldsymbol{u}_{\text {hom }}$, which is a unique solution in $\mathcal{H}_{\text {per }}(\mathbf{c u r l}, \mathbf{T})$ of the homogenised problem:

$$
\operatorname{curl}\left(\varepsilon_{\text {hom }}^{-1}(\boldsymbol{x}) \operatorname{curl} \boldsymbol{u}_{\mathrm{hom}}\right)+\boldsymbol{u}_{\mathrm{hom}}=\boldsymbol{f},
$$

where the effective matrix $\varepsilon_{\text {hom }}(\boldsymbol{x})$ is given by ${ }^{3}$

$$
\varepsilon_{\mathrm{hom}}(\boldsymbol{x}) \boldsymbol{\xi} \cdot \boldsymbol{\xi}=\inf _{\Phi \in H_{\mathrm{per}}^{1}(Y)} \int_{Y} \varepsilon(\boldsymbol{x}, \boldsymbol{y})(\boldsymbol{\xi}+\nabla \Phi(\boldsymbol{y})) \cdot(\boldsymbol{\xi}+\nabla \Phi(\boldsymbol{y})) \mathrm{d} \boldsymbol{y}
$$

for any $\boldsymbol{\xi} \in \mathbb{R}^{3}$.

Further, as $\eta \rightarrow 0$, the restrictions to $L_{\mathrm{sol}}^{2}(\mathbf{T})$ of the operators $\mathcal{G}_{\eta}$ converge in norm to the restriction to $L_{\text {sol }}^{2}(\mathbf{T})$ of the limit operator $\mathcal{G}$ defined for every $\boldsymbol{f} \in \mathbf{L}^{2}(\mathbf{T})$ by $\mathcal{G} \boldsymbol{f}=\boldsymbol{u}_{\text {hom }}$, where $\boldsymbol{u}_{\text {hom }}$ is the solution of (1.3). This fact follows from the weak compactness of the unit ball in $\mathbf{L}^{2}(\mathbf{T})$ and the above mentioned result that when a sequence of $\boldsymbol{f}_{\eta} \in \mathbf{L}_{\text {sol }}^{2}(\mathbf{T})$ weakly converges to $f \in \mathbf{L}_{\text {sol }}^{2}(\mathbf{T})$ one has the strong convergence in $\mathbf{L}^{2}(\mathbf{T})$ of $\mathcal{G}_{\eta} \boldsymbol{f}_{\eta}$ to $\mathcal{G} \boldsymbol{f}$. [The proof of an analogous statement can be found in [3, Theorem 2.2].] Clearly, $\mathcal{G}$ is a compact self-adjoint operator and one has $\sigma(\mathcal{G})=\{0,1\} \bigcup\left\{\tilde{\Lambda}^{k}\right\}_{k \geq 1}$, where the eigenvalues $\tilde{\Lambda}^{k}$ are listed in the decreasing order and $\lim _{k \rightarrow+\infty} \tilde{\Lambda}^{k}=0$. The min-max principle (see e.g. [7]) implies that $\left|\tilde{\Lambda}_{\eta}^{k}-\tilde{\Lambda}^{k}\right| \leq\left\|\mathcal{G}_{\eta}-\mathcal{G}\right\|$. Therefore, the above convergence in norm yields the convergence of each individual eigenvalue $\tilde{\Lambda}_{\eta}^{k}$ labelled in the decreasing order, and therefore of each eigenvalue $\Lambda_{\eta}^{k}$ of (1.1). In particular, when $\eta \rightarrow 0$, the set

\footnotetext{
${ }^{1}$ The space $\mathcal{H}_{\text {per }}(\mathbf{c u r l}, \mathbf{T})$ is defined as the closure of the set $\mathbf{C}_{\mathrm{per}}^{\infty}(\mathbf{T})$ of $\mathbf{T}$-periodic infinitely smooth vector functions $\boldsymbol{\varphi}$ with respect to the norm $\|\boldsymbol{\varphi}\|_{\mathbf{L}^{2}(\mathbf{T})}+\|\operatorname{curl} \boldsymbol{\varphi}\|_{\mathbf{L}^{2}(\mathbf{T})}$.

${ }^{2}$ The space $\mathbf{L}_{\text {sol }}^{2}(\mathbf{T})$ is defined to consist of those functions in $\mathbf{L}^{2}(\mathbf{T})$ whose divergence vanishes, with the Hilbert structure induced from $\mathbf{L}(\mathbf{T})$. Denote by $H_{\mathrm{per}}^{1}(\mathbf{T})$ the closure of $C_{\mathrm{per}}^{\infty}(\mathbf{T})$ in the norm $\|\varphi\|_{L^{2}(\mathbf{T})}+\|\nabla \varphi\|_{\mathbf{L}^{2}(\mathbf{T})}$. Then the divergence div $\boldsymbol{u}$ of a vector function $\boldsymbol{u} \in \mathbf{L}^{2}(\mathbf{T})$ is a functional on $H_{\mathrm{per}}^{1}(\mathbf{T})$ defined by the formula $\langle\operatorname{div} \boldsymbol{u}, \varphi\rangle=-\int_{\mathbf{T}} \boldsymbol{u}(\boldsymbol{x}) \cdot \nabla \varphi(\boldsymbol{x}) \mathrm{d} \boldsymbol{x}$.

${ }^{3}$ This representation is consistent with the classical homogenisation result in two dimensions for a sequence $\boldsymbol{u}_{\eta}=u_{\eta}^{3}\left(x_{1}, x_{2}\right) \boldsymbol{e}_{3}$ (transverse electric polarisation), involving the inverse of the matrix $\varepsilon(\boldsymbol{x}, \boldsymbol{y})$ in the definition (1.5), see [18]. This can be obtained by examining the dual form of (1.5) associated to $\varepsilon_{\text {hom }}^{-1}(\boldsymbol{x})$ :
}

$$
\varepsilon_{\mathrm{hom}}^{-1}(\boldsymbol{x}) \boldsymbol{\xi} \cdot \boldsymbol{\xi}=\inf _{\Psi \in \mathcal{H}_{\mathrm{per}}(\operatorname{curl}, Y)} \int_{Y} \varepsilon^{-1}(\boldsymbol{x}, \boldsymbol{y})(\boldsymbol{\xi}+\operatorname{curl} \Psi(\boldsymbol{y})) \cdot(\boldsymbol{\xi}+\operatorname{curl} \boldsymbol{\Psi}(\boldsymbol{y})) \mathrm{d} \boldsymbol{y} .
$$


$\sigma_{\eta}$ converges (in the Hausdorff sense ${ }^{4}$ ) to $\left\{(1-\Lambda)^{-1} \Lambda: \Lambda \in \sigma(\mathcal{G})\right\}$. However, this convergence result alone does not say anything about the asymptotic behaviour of sequences of eigenvalues $\Lambda_{\eta}^{k(\eta)}$ when $\eta \rightarrow 0$, where $k(\eta) \rightarrow \infty$ (which corresponds to high frequencies $\Lambda_{\eta}^{-1}$ ), and this is the issue that we address in the present work. It turns out that the behaviour of the eigenvalues $\Lambda_{\eta}$ as $\eta \rightarrow 0$ depends on the way they are scaled with $\eta$. In particular, three different limiting operators can be constructed, depending on whether the limit of $\eta^{-2} \Lambda_{\eta}$ is finite, infinite, or zero. The first case, which is of greatest interest in applications, corresponds to a limiting operator with a band-gap spectrum.

We now outline the plan of the paper. In Section 2, we recall the concept of two-scale convergence for sequences in $\mathbf{L}^{2}(\mathbf{T})$ and adapt it to sequences of bounded self-adjoint operators. In Sections 3 and 4 , we characterise the limit spectrum of the type $\lim _{\eta \rightarrow 0} \eta^{-2} \sigma_{\eta}$. Following [3], we will study the eigenvalues of order $\eta^{2}$. Via a change of spectral parameter in (1.1), we look for pairs $\left(\mu_{\eta}, \boldsymbol{H}_{\eta}\right) \in \mathbb{R}^{+} \times \mathcal{H}_{\text {per }}(\mathbf{c u r l}, \mathbf{T})$, $\boldsymbol{H}_{\eta} \not \equiv 0$, such that

$$
\eta^{2} \operatorname{curl}\left(\varepsilon^{-1}(\boldsymbol{x}, \boldsymbol{x} / \eta) \operatorname{curl} \boldsymbol{H}_{\eta}\right)+\boldsymbol{H}_{\eta}=\mu_{\eta}^{-1} \boldsymbol{H}_{\eta} .
$$

This transformation of (1.1) into (1.6) keeps unchanged the eigenfunctions and changes the eigenvalues $\Lambda_{\eta}$ to $\mu_{\eta}=\left(\eta^{2}+\Lambda_{\eta}\right)^{-1} \Lambda_{\eta}$, ensuring that $\mu_{\eta} \sim 1$ if $\Lambda_{\eta} \sim \eta^{2}$.

The problem (1.6) generates an operator $\mathcal{S}_{\eta} \in \mathcal{L}\left(\mathbf{L}^{2}(\mathbf{T})\right)$ defined for each $\boldsymbol{f} \in \mathbf{L}^{2}(\mathbf{T})$ by $\mathcal{S}_{\eta} \boldsymbol{f}=\boldsymbol{v}_{\eta}$, where $\boldsymbol{v}_{\eta}$ is the unique solution in $\mathcal{H}_{\text {per }}(\mathbf{c u r l}, \mathbf{T})$ of the problem

$$
\eta^{2} \operatorname{curl}\left(\varepsilon^{-1}(\boldsymbol{x}, \boldsymbol{x} / \eta) \operatorname{curl} \boldsymbol{v}_{\eta}\right)+\boldsymbol{v}_{\eta}=\boldsymbol{f} .
$$

It can be shown that $\mathcal{S}_{\eta}$ converge weakly to the identity operator, but this fact does not provide any information on the behaviour of the spectra of $\mathcal{S}_{\eta}$. The latter issue can be handled using the notion of twoscale convergence [1], whereby the limit operator $\mathcal{S}^{K}$ defined for every $K \in \mathbb{N}$ acts on the space $\mathbf{L}^{2}(\mathbf{T} \times K Y)$. The (renormalised) limit of $\sigma_{\eta}$ will then be determined by studying the spectra $\sigma\left(\mathcal{S}^{K}\right), K \in \mathbb{N}$, via the Bloch wave decomposition. For this, we introduce a family of limit problems, whereby for every $(\boldsymbol{x}, \boldsymbol{\theta}) \in \mathbf{T} \times Y$, we look for pairs $(\mu(\boldsymbol{x}, \boldsymbol{\theta}), \boldsymbol{v}(\boldsymbol{y})) \in \mathbb{R}^{+} \times \mathcal{H}_{\text {per }}(\mathbf{c u r l}, Y)$ satisfying the equation

$$
\operatorname{curl}_{y}\left(\varepsilon^{-1}(\boldsymbol{x}, \boldsymbol{y}) \operatorname{curl}_{y}\left(\boldsymbol{v}(\boldsymbol{y}) e^{2 \pi i \boldsymbol{\theta} \cdot \boldsymbol{y}}\right)\right)+\boldsymbol{v}(\boldsymbol{y}) e^{2 \pi i \boldsymbol{\theta} \cdot \boldsymbol{y}}=\mu(\boldsymbol{x}, \boldsymbol{\theta})^{-1} \boldsymbol{v}(\boldsymbol{y}) e^{2 \pi i \boldsymbol{\theta} \cdot \boldsymbol{y}} .
$$

For the spectrum $\sigma(\boldsymbol{x}, \boldsymbol{\theta})$ of the problem (1.7) we have $\sigma(\boldsymbol{x}, \boldsymbol{\theta})=\{0,1\} \bigcup\left\{\mu^{k}(\boldsymbol{x}, \boldsymbol{\theta})\right\}_{k \geq 1}$, where the sequence $\mu^{k}(\boldsymbol{x}, \boldsymbol{\theta})$ converges to 0 . It can be shown that for each fixed $k$, the eigenvalue $\mu^{k}(\boldsymbol{x}, \boldsymbol{\theta})$ is a continuous function of $(\boldsymbol{x}, \boldsymbol{\theta})$ ( $c f .[3])$. We then define the Bloch spectrum (band spectrum) by

$$
\sigma_{\text {Bloch }}:=\{0,1\} \cup \bigcup_{k \geq 1}\left[\min _{(x, \theta) \in \mathbf{T} \times Y} \mu^{k}(\boldsymbol{x}, \boldsymbol{\theta}), \max _{(x, \theta) \in \mathbf{T} \times Y} \mu^{k}(\boldsymbol{x}, \boldsymbol{\theta})\right]
$$

Note that $\sigma_{\text {Bloch }}$ is the spectrum of a non-compact operator that maps every vector-function $\boldsymbol{f}(\boldsymbol{x}, \boldsymbol{y}) \in$ $\mathbf{L}^{2}(\mathbf{T} \times Y)$ to the solution $\boldsymbol{w}(\boldsymbol{x}, \boldsymbol{y}) \in \mathbf{L}^{2}\left(\mathbf{T}, \mathcal{H}_{\text {per }}(\mathbf{c u r l}, Y)\right)$ of

$$
\operatorname{curl}_{y}\left(\varepsilon^{-1}(\boldsymbol{x}, \boldsymbol{y}) \operatorname{curl}_{y} \boldsymbol{w}(\boldsymbol{x}, \boldsymbol{y})\right)+\boldsymbol{w}(\boldsymbol{x}, \boldsymbol{y})=\boldsymbol{f}(\boldsymbol{x}, \boldsymbol{y}) \text { a.e. } \boldsymbol{x} \in \mathbf{T} .
$$

In Section 5 we characterise the limit spectrum for other scalings. An appropriate multi-scale generalisation of the two-scale convergence method [5] is used to study the spectra of the types $a_{\eta}^{-2} \sigma_{\eta}$, where $a_{\eta} \gg \eta$ or $a_{\eta} \ll \eta$. Section 6 is devoted to numerical illustration of the spectrum convergence result in the "critical" case $a_{\eta} \sim \eta$. Finally, the Appendix contains some of the technical material related to the results of the paper.

\section{Two-scale convergence for operators}

The notion of two-scale convergence was introduced by Nguetseng and Allaire (see [12], [1]) for sequences of bounded vector functions in $\mathbf{L}^{2}$-norm. In what follows, we recall its definition, some of it basic properties and adapt it to sequences of bounded self-adjoint operators, see also [8].

Definition 1. Consider a bounded set $\mathbf{T} \subset \mathbb{R}^{N}$ and an integer $K \in \mathbb{N}$.

i) A vector function $\boldsymbol{\varphi} \in \mathbf{L}^{2}(\mathbf{T} \times K Y)$ is said to be admissible if the functions $\boldsymbol{\varphi}_{\eta}(\boldsymbol{x})=\boldsymbol{\varphi}(\boldsymbol{x}, \boldsymbol{x} / \eta)$, $\eta \in(0,1]$, are uniformly bounded in $\mathbf{L}^{2}(\mathbf{T})$ and satisfy the condition

$$
\lim _{\eta \rightarrow 0} \int_{\mathbf{T}}\left|\boldsymbol{\varphi}_{\eta}(\boldsymbol{x})\right|^{2} \mathrm{~d} \boldsymbol{x}=\frac{1}{K^{N}} \int_{\mathbf{T}} \int_{K Y}|\boldsymbol{\varphi}(\boldsymbol{x}, \boldsymbol{y})|^{2} \mathrm{~d} \boldsymbol{y} \mathrm{d} \boldsymbol{x} .
$$

\footnotetext{
${ }^{4}$ We say that $\sigma_{\eta}$ converges to $\sigma$ in the Hausdorff sense if $\max \left\{\sup _{\lambda_{1} \in \sigma_{\eta}} \inf _{\lambda_{2} \in \sigma}\left|\lambda_{1}-\lambda_{2}\right|, \sup _{\lambda_{2} \in \sigma} \inf _{\lambda_{1} \in \sigma_{\eta}}\left|\lambda_{1}-\lambda_{2}\right|\right\} \rightarrow 0$ as $\eta \rightarrow 0$.
} 
ii) Denote by $\eta$ a sequence of positive numbers converging to zero. A sequence $\boldsymbol{u}_{\eta}$ of functions in $\mathbf{L}^{2}(\mathbf{T})$ is said to weakly two-scale converge to $\boldsymbol{u}_{0}(\boldsymbol{x}, \boldsymbol{y}) \in \mathbf{L}^{2}(\mathbf{T} \times K Y)$, in which case we write $\boldsymbol{u}_{\eta} \rightarrow \boldsymbol{u}_{0}$, if for any admissible $\boldsymbol{\psi}$ e.g. $\boldsymbol{\psi}(\boldsymbol{x}, \boldsymbol{y}) \in \mathbf{C}\left(\mathbf{T}, C_{\mathrm{per}}^{\infty}(K Y)\right)$, one has

$$
\lim _{\eta \rightarrow 0} \int_{\mathbf{T}} \boldsymbol{u}(\boldsymbol{x}) \cdot \boldsymbol{\psi}(\boldsymbol{x}, \boldsymbol{x} / \eta) \mathrm{d} \boldsymbol{x}=\frac{1}{K^{N}} \int_{\mathbf{T}} \int_{K Y} \boldsymbol{u}_{0}(\boldsymbol{x}, \boldsymbol{y}) \cdot \boldsymbol{\psi}(\boldsymbol{x}, \boldsymbol{y}) \mathrm{d} \boldsymbol{y} \mathrm{d} \boldsymbol{x} .
$$

iii) A sequence $\boldsymbol{u}_{\eta}$ of functions in $\mathbf{L}^{2}(\mathbf{T})$ is said to strongly two-scale converge to $\boldsymbol{u}_{0}(\boldsymbol{x}, \boldsymbol{y}) \in \mathbf{L}^{2}(\mathbf{T} \times K Y)$, and we write $\boldsymbol{u}_{\eta} \rightarrow \boldsymbol{u}_{0}$, if any of the two equivalent statements holds:

a)

$$
\boldsymbol{u}_{\eta} \rightarrow \boldsymbol{u}_{0} \text { and } \lim _{\eta \rightarrow 0} \int_{\mathbf{T}}\left|\boldsymbol{u}_{\eta}(\boldsymbol{x})\right|^{2} \mathrm{~d} \boldsymbol{x}=\frac{1}{K^{N}} \int_{\mathbf{T}} \int_{K Y}\left|\boldsymbol{u}_{0}(\boldsymbol{x}, \boldsymbol{y})\right|^{2} \mathrm{~d} \boldsymbol{y} \mathrm{d} \boldsymbol{x}
$$

b) For any sequence $\boldsymbol{v}_{\eta}$ such that $\boldsymbol{v}_{\eta} \rightarrow \boldsymbol{v} \in \mathbf{L}^{2}(\mathbf{T})$,

$$
\int_{\mathbf{T}} \boldsymbol{u}_{\eta}(\boldsymbol{x}) \cdot \boldsymbol{v}_{\eta}(\boldsymbol{x}) \mathrm{d} \boldsymbol{x} \stackrel{\eta \rightarrow 0}{\longrightarrow} \frac{1}{K^{N}} \int_{\mathbf{T}} \int_{K Y} \boldsymbol{u}_{0}(\boldsymbol{x}, \boldsymbol{y}) \cdot \boldsymbol{v}(\boldsymbol{x}, \boldsymbol{y}) \mathrm{d} \boldsymbol{y} \mathrm{d} \boldsymbol{x} .
$$

iv) A sequence $\mathcal{S}_{\eta}$ of operators in $\mathbf{L}^{2}(\mathbf{T})$ is said to strongly two-scale converge to an operator $\mathcal{S}$ in $\left.\mathbf{L}^{2}(\mathbf{T} \times K Y)\right)$ if for any sequence $\boldsymbol{f}_{\eta} \in \mathbf{L}^{2}(\mathbf{T})$ such that $\boldsymbol{f}_{\eta} \rightarrow \boldsymbol{f} \in \mathbf{L}^{2}(\mathbf{T} \times K Y)$ one has $\mathcal{S}_{\eta} \boldsymbol{f}_{\eta} \rightarrow \mathcal{S} \boldsymbol{f}$.

The crucial property of the two-scale convergence that makes it useful in studying elliptic PDEs is the compactness of bounded sets in $\mathbf{L}^{2}(\mathbf{T})$, see e.g. [1]. The usual argument therefore is to establish a bound on a solution sequence and then infer an equation on the limit of its subsequence. The notion of two-scale operator convergence, in turn, allows one to simplify the derivation of the associated spectral convergence. In fact, given a sequence of bounded linear self-adjoint operators $\mathcal{S}_{\eta}$, one can state the following result on strong two-scale convergence for the families of the associated spectral projections $\left\{E_{\lambda}\left(\mathcal{S}_{\eta}\right)\right\}_{\lambda \in \mathbb{R}}(c f$. [8]). We recall that for a given operator $\mathcal{A}$, the function $E_{\lambda}(\mathcal{A})$ is non-decreasing, upper semi-continuous, non-negative, and satisfies the identity $E_{\lambda}(\mathcal{A}) E_{\mu}(\mathcal{A})=E_{\min \{\lambda, \mu\}}(\mathcal{A})$ for any $\lambda, \mu \in \mathbb{R}$ (see e.g. [7], [14]).

Lemma 1. Let $\mathcal{S}_{\eta}$ be a sequence of bounded linear self-adjoint operators in $\mathbf{L}^{2}(\mathbf{T})$ that strongly two-scale converges to an operator $\mathcal{S}$ in $\mathbf{L}^{2}(\mathbf{T} \times K Y)$. Then $\mathcal{S}$ is linear and self-adjoint, and for any $\mu \in \mathbb{R}$ that is not an eigenvalue of $\mathcal{S}$, one has $E_{\mu}\left(\mathcal{S}_{\eta}\right) \rightarrow E_{\mu}(\mathcal{S})$ as $\eta \rightarrow 0$.

Proof. Without loss of generality, we can assume that $\mu=0$, for otherwise, the sequence $\mathcal{S}_{\eta}-\mu I$ can be considered. The linearity and self-adjointness of $\mathcal{S}$ is clear from that of $\mathcal{S}_{\eta}$ and the fact that $\mathcal{S}_{\eta}$ two-scale converge to $\mathcal{S}$. Further, for any sequence $\boldsymbol{f}_{\eta} \in \mathbf{L}^{2}(\mathbf{T})$ such that $\boldsymbol{f}_{\eta} \rightarrow \boldsymbol{f} \in \mathbf{L}^{2}(\mathbf{T} \times K Y)$ we have $\mathcal{S}_{\eta} \boldsymbol{f}_{\eta} \rightarrow \mathcal{S} \boldsymbol{f}$ and $\mathcal{S}_{\eta}^{2} \boldsymbol{f}_{\eta} \rightarrow \mathcal{S}^{2} \boldsymbol{f}$. It is clear that by iterating this procedure we prove that for any polynomial $P$ the convergence $P\left(\mathcal{S}_{\eta}\right) \rightarrow P(\mathcal{S})$ holds as $\eta \rightarrow 0$.

Since the operators $\mathcal{S}_{\eta}, \eta \in \Xi$, are bounded, and for any $\boldsymbol{f} \in \mathbf{L}^{2}(\mathbf{T}), \mathcal{S}_{\eta} \boldsymbol{f} \rightarrow \mathcal{S} \boldsymbol{f}$ in $\mathbf{L}^{2}(\mathbf{T})$, by the BanachSteinhaus theorem (see e.g. [7], [15]) $\mathcal{S}_{\eta}$ are uniformly bounded. Hence, there exists a constant $C>0$ such that $\sigma_{\eta} \subset[-C, C]$ for any $\eta \in(0,1]$, and by the spectral theorem the operators $\mathcal{S}_{\eta}, \eta \in(0,1]$, and $\mathcal{S}$ can be composed with any continuous function $F$ on $[-C, C]$. Notice that for any polynomial $P$ and a sequence $\boldsymbol{f}_{\eta} \in \mathbf{L}^{2}(\mathbf{T}), \boldsymbol{f}_{\eta} \rightarrow \boldsymbol{f} \in \mathbf{L}^{2}(\mathbf{T} \times K Y)$, we have

$$
F\left(\mathcal{S}_{\eta}\right) \boldsymbol{f}_{\eta}-F(\mathcal{S}) \boldsymbol{f}=\left(F\left(\mathcal{S}_{\eta}\right) \boldsymbol{f}_{\eta}-P\left(\mathcal{S}_{\eta}\right) \boldsymbol{f}_{\eta}\right)+\left(P\left(\mathcal{S}_{\eta}\right) \boldsymbol{f}_{\eta}-P(\mathcal{S}) \boldsymbol{f}\right)+(P(\mathcal{S}) \boldsymbol{f}-F(\mathcal{S}) \boldsymbol{f}) .
$$

Further, there exists $c>0$ such that

$$
\left\|F\left(\mathcal{S}_{\eta}\right) \boldsymbol{f}_{\eta}-P\left(\mathcal{S}_{\eta}\right) \boldsymbol{f}_{\eta}\right\|_{\mathbf{L}^{2}(\mathbf{T})} \leq\left\|\boldsymbol{f}_{\eta}\right\|_{\mathbf{L}^{2}(\mathbf{T})} \sup _{s \in[-C, C]}|F(s)-P(s)| \leq c\|F-P\|_{L^{\infty}([-C, C])},
$$

and

$$
\left\|P(\mathcal{S}) f-F\left(\mathcal{S}_{\eta}\right) f\right\|_{\mathbf{L}^{2}(\mathbf{T})} \leq\|f\|_{\mathbf{L}^{2}(\mathbf{T} \times K Y)}\|P(\mathcal{S})-F(\mathcal{S})\|_{\mathcal{L}\left(\mathbf{L}^{2}(\mathbf{T} \times K Y)\right)} \leq\|f\|_{\mathbf{L}^{2}(\mathbf{T} \times K Y)}\|F-P\|_{L^{\infty}([-C, C])} .
$$

By the Weierstrass theorem there is a sequence of polynomials $P_{n}$ such that $\left\|F-P_{n}\right\|_{L^{\infty}([-C, C])} \rightarrow 0$. Hence we deduce that

$$
F\left(\mathcal{S}_{\eta}\right) \boldsymbol{f}_{\eta} \rightarrow F(\mathcal{S}) \boldsymbol{f}
$$

Now, consider a continuous function $G$ on $\mathbb{R}$ such that $G(\lambda)=1$ when $\lambda \leq 0, G(\lambda)=0$ when $\lambda \geq 1$ and $|G(\lambda)| \leq 1$ for $\lambda \in[0,1]$. For any $n \in \mathbb{N}$, the spectral theorem (see e.g. [7], [14]) implies ${ }^{5}$

$$
G\left(n \mathcal{S}_{\eta}\right) \boldsymbol{f}_{\eta}=\int_{-\infty}^{\infty} G(n \lambda) d E_{\lambda}\left(\mathcal{S}_{\eta}\right) \boldsymbol{f}_{\eta}=\int_{-\infty}^{0} d E_{\lambda}\left(\mathcal{S}_{\eta}\right) \boldsymbol{f}_{\eta}+\left(E_{1 / n}\left(\mathcal{S}_{\eta}\right)-E_{0}\left(\mathcal{S}_{\eta}\right)\right) \boldsymbol{f}_{\eta} .
$$

\footnotetext{
${ }^{5}$ The integrals with respect to the spectral projections are understood in the sense of Stieltjes, see e.g. [15].
} 
We claim that the last term in (2.11) converges to zero strongly in $\mathbf{L}^{2}(\mathbf{T})$ as $n \rightarrow \infty$, and the convergence is uniform in $\eta$. Indeed, consider a continuous function $\psi$ on $\mathbb{R}$ such that $\psi(\lambda)=1$ when $0 \leq \lambda \leq 1$, $\operatorname{supp} \psi \subset[-1,2]$, and $\psi(\lambda) \geq 0$ for any $\lambda \in \mathbb{R}$. Then for any $\delta>0$ and any subsequence $\eta_{n}$ one has

$$
\begin{gathered}
\left\langle\int_{-\infty}^{\infty} \psi\left(\delta^{-1} \lambda\right) d E_{\lambda}(\mathcal{S}) \boldsymbol{f}, \boldsymbol{f}\right\rangle=\lim _{n \rightarrow \infty}\left\langle\int_{-\infty}^{\infty} \psi\left(\delta^{-1} \lambda\right) d E_{\lambda}\left(\mathcal{S}_{\eta_{n}}\right) \boldsymbol{f}_{\eta_{n}}, \boldsymbol{f}_{\eta_{n}}\right\rangle \\
\geq \lim _{n \rightarrow \infty}\left\langle\int_{0}^{1 / n} d E_{\lambda}\left(\mathcal{S}_{\eta_{n}}\right) \boldsymbol{f}_{\eta_{n}}, \boldsymbol{f}_{\eta_{n}}\right\rangle=\lim _{n \rightarrow \infty}\left\|\left(E_{1 / n}\left(\mathcal{S}_{\eta_{n}}\right)-E_{0}\left(\mathcal{S}_{\eta_{n}}\right)\right) \boldsymbol{f}_{\eta_{n}}\right\|_{\mathbf{L}^{2}(\mathbf{T})}^{2},
\end{gathered}
$$

where the angle brackets denote the usual scalar products in $\mathbf{L}^{2}(\mathbf{T} \times K Y)$ and $\mathbf{L}^{2}(\mathbf{T})$, and the first expression tends to zero as $\delta \rightarrow 0$ in view of the assumption that $\mu=0$ is not an eigenvalue of $\mathcal{S}$. Since the subsequence $\eta_{n}$ was arbitrary, the above claim follows. Combining it with the straightforward observation that $G(n \mathcal{S}) \boldsymbol{f}$ converge in $\mathbf{L}^{2}(\mathbf{T})$ to $E_{0}(\mathcal{S}) \boldsymbol{f}$ as $n \rightarrow \infty$ and invoking $(2.10)$ with $F(\cdot)=G(n \cdot)$ we conclude that the convergence $E_{0}\left(\mathcal{S}_{\eta}\right) \boldsymbol{f}_{\eta} \rightarrow E_{0}(\mathcal{S}) \boldsymbol{f}$ holds.

The convergence of spectral families gives little information about the pointwise convergence of the spectra. As an alternative to the above lemma we have the following result.

Lemma 2. Consider a family of operators $\mathcal{S}_{\eta} \in \mathcal{L}\left(\mathbf{L}^{2}(\mathbf{T})\right)$ and an operator $\mathcal{S} \in \mathcal{L}\left(\mathbf{L}^{2}(\mathbf{T} \times K Y)\right)$. If $S_{\eta} \rightarrow S$ then for any $\mu \in \sigma(\mathcal{S})$ there exists a sequence $\mu_{\eta} \in \sigma\left(\mathcal{S}_{\eta}\right)$ such that $\mu_{\eta} \rightarrow \mu$ as $\eta \rightarrow 0$.

Proof. Suppose the opposite is true, i.e. $\mu \in \sigma(\mathcal{S})$ and there exists $\delta>0$ such that for any sequence $\mu_{\eta} \in \sigma\left(\mathcal{S}_{\eta}\right)$ one has $\left|\mu_{\eta}-\mu\right|>\delta$. For any $\boldsymbol{f} \in \mathbf{C}_{\text {per }}^{\infty}(\mathbf{T} \times K Y)$ we can then write

$$
\begin{aligned}
& \delta\|\boldsymbol{f}(\boldsymbol{x}, \boldsymbol{x} / \eta)\|_{\mathbf{L}^{2}(\mathbf{T})} \leq\left\|\mathcal{S}_{\eta} \boldsymbol{f}(\boldsymbol{x}, \boldsymbol{x} / \eta)-\mu \boldsymbol{f}(\boldsymbol{x}, \boldsymbol{x} / \eta)\right\|_{\mathbf{L}^{2}(\mathbf{T})} \\
= & \sup _{\varphi \in \mathbf{C}_{\mathrm{per}}^{\infty}(\mathbf{T} \times K Y)} \int_{\mathbf{T}}\left(\mathcal{S}_{\eta} \boldsymbol{f}(\boldsymbol{x}, \boldsymbol{x} / \eta)-\mu \boldsymbol{f}(\boldsymbol{x}, \boldsymbol{x} / \eta)\right) \cdot \boldsymbol{\varphi}(\boldsymbol{x}, \boldsymbol{x} / \eta) \mathrm{d} \boldsymbol{x} .
\end{aligned}
$$

Passing to the two-scale limit as $\eta \rightarrow 0$ in the above inequality yields

$$
\delta\|\boldsymbol{f}\|_{\mathbf{L}^{2}(\mathbf{T} \times K Y)} \leq \sup _{\varphi \in \mathbf{C}_{\text {per }}^{\infty}(\mathbf{T} \times K Y)} \int_{\mathbf{T}} \int_{K Y}(\mathcal{S} \boldsymbol{f}(\boldsymbol{x}, \boldsymbol{y})-\mu \boldsymbol{f}(\boldsymbol{x}, \boldsymbol{y})) \cdot \boldsymbol{\varphi}(\boldsymbol{x}, \boldsymbol{y}) \mathrm{d} \boldsymbol{y} \mathrm{d} \boldsymbol{x}=\|\mathcal{S} \boldsymbol{f}-\mu \boldsymbol{f}\|_{\mathbf{L}^{2}(\mathbf{T} \times K Y)} .
$$

By the density of $\mathbf{C}_{\text {per }}^{\infty}(\mathbf{T} \times K Y)$ in $\mathbf{L}^{2}(\mathbf{T} \times K Y)$ and the boundedness of $\mathcal{S}$ we obtain the inequality (2.12) for any $\boldsymbol{f} \in \mathbf{L}^{2}(\mathbf{T} \times K Y)$, which is a contradiction with the assumption that $\mu \in \sigma(\mathcal{S})$.

\section{Bloch-wave homogenisation}

In this section we prove the convergence of spectral families associated to sequences of eigenvalues of the order $\eta^{2}$ for the problem (1.1). The physical application of this model is based on the property of the possible appearance of photonic band gaps in the spectrum, i.e. a range of frequencies for which no wave can propagate within the medium. This result is based on the following statement.

Theorem 1. Consider the domain $\mathbf{T}=[-T, T]^{3}$, and $\eta \in \Xi$. We look for $\boldsymbol{u}_{\eta} \in \mathcal{H}_{\text {per }}(\mathbf{c u r l}, \mathbf{T})$ such that

$$
\eta^{2} \operatorname{curl}\left(\varepsilon^{-1}(\boldsymbol{x}, \boldsymbol{x} / \eta) \operatorname{curl} \boldsymbol{u}_{\eta}\right)+\boldsymbol{u}_{\eta}=\boldsymbol{f}_{\eta}
$$

with a coercive matrix $\varepsilon(\boldsymbol{x}, \boldsymbol{y})$ whose entries are in $C_{\mathrm{per}}\left(\mathbf{T}, L^{\infty}(Y)\right)$, and $\boldsymbol{f}_{\eta} \in \mathbf{L}^{2}(\mathbf{T})$.

i) The solution of (3.13) exists and is unique in $\mathcal{H}_{\text {per }}(\mathbf{c u r l}, \mathbf{T})$.

ii) Suppose that $K \in \mathbb{N}$ and $\boldsymbol{f}_{\eta} \rightarrow \boldsymbol{f} \in \mathbf{L}^{2}(\mathbf{T} \times K Y)$. Then there is a subsequence of problems (3.13), for which we keep the same index $\eta$, such that $\boldsymbol{u}_{\eta} \rightarrow \boldsymbol{u}_{0}$ and $\eta \mathbf{c u r l} \boldsymbol{u}_{\eta} \rightarrow \operatorname{curl}_{y} \boldsymbol{u}_{0}$, where $\boldsymbol{u}_{0} \in$ $L^{2}\left(\mathbf{T}, \mathcal{H}_{\text {per }}(\mathbf{c u r l}, K Y)\right)$ and for almost every $\boldsymbol{x} \in \mathbf{T}$ the function $\boldsymbol{u}_{0}(\boldsymbol{x}, \cdot)$ is a solution of

$$
\operatorname{curl}_{y}\left(\varepsilon^{-1}(\boldsymbol{x}, \boldsymbol{y}) \operatorname{curl}_{y} \boldsymbol{u}_{0}\right)+\boldsymbol{u}_{0}=\boldsymbol{f}(\boldsymbol{x}, \cdot)
$$

Proof. See the Appendix.

Remark 1. It can be shown via a standard argument that the convergences $\boldsymbol{u}_{\eta} \rightarrow \boldsymbol{u}_{0}$ and $\eta \mathbf{c u r l} \boldsymbol{u}_{\eta} \rightarrow$ $\operatorname{curl}_{y} \boldsymbol{u}_{0}$, hold for the whole original sequence of indices $\eta$, not just for a subsequence. 
Corollary 1. Let $\mathcal{S}_{\eta}$ be a sequence of operators defined on the space $\mathbf{L}^{2}(\mathbf{T})$ by $\mathcal{S}_{\eta} \boldsymbol{f}=\boldsymbol{u}_{\eta}$, where $\boldsymbol{u}_{\eta} \in$ $\mathcal{H}_{\text {per }}(\mathbf{c u r l}, \mathbf{T})$ is the solution of (3.13). Then

i) For every $K \in \mathbb{N}$ the strong two-scale convergence $\mathcal{S}_{\eta} \rightarrow \mathcal{S}^{K}$ holds. Here $\mathcal{S}^{K}$ is defined on $\mathbf{L}^{2}(\mathbf{T} \times K Y)$ by the formula $\mathcal{S}^{K} \boldsymbol{f}=\boldsymbol{u}_{0}$, where $\boldsymbol{u}_{0} \in L^{2}\left(\mathbf{T}, \mathcal{H}_{\text {per }}(\mathbf{c u r l}, K Y)\right)$ is the unique solution of (3.14).

ii) If $\mu \in \mathbb{R}$ is not an eigenvalue of $\mathcal{S}^{K}$, then $E_{\mu}\left(\mathcal{S}_{\eta}\right) \rightarrow E_{\mu}\left(\mathcal{S}^{K}\right)$, and for any $K \in \mathbb{N}$ the inclusion $\sigma\left(\mathcal{S}^{K}\right) \subset \lim _{\eta \rightarrow 0} \sigma\left(\mathcal{S}_{\eta}\right)$ holds.

Proof. The part i) follows form the fact that $\boldsymbol{u}_{\eta} \rightarrow \boldsymbol{u}$ and the definition of the strong two-scale convergence of operators. The part ii) is then an immediate consequence of Lemma 1 and Lemma 2.

The above corollary provides only a part of the proof of the spectral convergence, namely the statement that all Bloch eigenvalues are included in the limiting (rescaled) spectrum. Indeed, the convergence of $\mathcal{S}_{\eta}$ to $\mathcal{S}^{K}$ cannot be uniform, for $\mathcal{S}_{\eta}$ is compact but $\mathcal{S}^{K}$ is not (cf. [3]). Hence, we can only deduce the lower semi-continuity of the spectrum i.e. that for any $K \in \mathbb{N}$ one has $\sigma\left(\mathcal{S}^{K}\right) \subset \lim _{\eta \rightarrow 0} \sigma\left(\mathcal{S}_{\eta}\right)$. In order to analyse the spectra of the operators $\mathcal{S}^{K}$, a discrete Bloch wave decomposition (see [7]) is used, which allows one to diagonalise $\mathcal{S}^{K}$ via a representation $\mathcal{S}^{K}=\mathcal{B}^{*} T^{K} \mathcal{B}$ where $\mathcal{B}$ is an isometry between $\mathbf{L}^{2}(\mathbf{T} \times K Y)$ and $\left[\mathbf{L}^{2}(\mathbf{T} \times Y)\right]^{K^{3}}$ defined by $\mathcal{B} \boldsymbol{u}=\left\{\hat{\boldsymbol{u}}_{j}\right\}$, when $\boldsymbol{u}(\boldsymbol{x}, \boldsymbol{y})=\sum_{0 \leq j \leq K-1} \hat{\boldsymbol{u}}_{j}(\boldsymbol{x}, \boldsymbol{y}) \exp \left(2 \mathrm{i} \pi K^{-1} \boldsymbol{j} \cdot \boldsymbol{y}\right)$, cf. [3]. As $K \rightarrow \infty$, the Bloch frequencies $K^{-1} \boldsymbol{j}$ are dense in $Y$ and it is natural to introduce $\boldsymbol{\theta} \in Y$ that parameterises the resolvent operators $T_{\theta, x} \in \mathcal{L}\left(\mathbf{L}^{2}(\mathbf{T})\right)$ associated with the problems (1.7), so that $T^{K}=\operatorname{diag}\left[T_{j^{-1} K}\right]_{0 \leq j \leq K-1}$, where for any $\boldsymbol{\theta} \in Y$, the operator $T_{\theta}$ maps $\boldsymbol{f} \in \mathbf{L}^{2}(\mathbf{T} \times Y)$ to the (unique) solution $\boldsymbol{u}(\boldsymbol{x}, \boldsymbol{y}) \in \mathcal{H}_{\text {per }}(\mathbf{c u r l}, \mathbf{T})$ of the equation $(c f .(1.7))$

$$
\operatorname{curl}_{y}\left(\varepsilon^{-1}(\boldsymbol{x}, \boldsymbol{y}) \operatorname{curl}_{y}\left(\boldsymbol{u}(\boldsymbol{x}, \boldsymbol{y}) e^{2 \pi i \boldsymbol{\theta} \cdot \boldsymbol{y}}\right)\right)+\boldsymbol{u}(\boldsymbol{x}, \boldsymbol{y}) e^{2 \pi i \boldsymbol{\theta} \cdot \boldsymbol{y}}=\boldsymbol{f}(\boldsymbol{x}, \boldsymbol{y}) e^{2 \pi i \boldsymbol{\theta} \cdot \boldsymbol{y}} .
$$

Finally, noting that the eigenvalues $\mu(\boldsymbol{x}, \boldsymbol{\theta})$ of $T_{\theta, x}$ are continuous (see e.g. [3] and references therein), we conclude that $\lim _{K \rightarrow \infty} \sigma\left(\mathcal{S}^{K}\right)=\bigcup_{\theta \in Y} \sigma\left(T_{\theta}\right)=\bigcup_{x \in \mathbf{T}, \theta \in Y} \sigma\left(T_{\theta, x}\right) \equiv \sigma_{\text {Bloch }}$, where $\sigma_{\text {Bloch }}$ is defined by (1.8). Hence, $\sigma_{\text {Bloch }} \subset \lim _{\eta \rightarrow 0} \sigma\left(\mathcal{S}_{\eta}\right)$. In the next section we show that in fact $\lim _{\eta \rightarrow 0} \sigma\left(\mathcal{S}_{\eta}\right) \backslash \sigma_{\text {Bloch }}=\emptyset$.

\section{Completeness of the limiting spectrum}

In order to substantiate the claim made at the end of the previous section, for any $\mu \in \sigma_{\text {Bloch }}$ we will: 1) Construct "approximate eigenfunctions" for the permittivity matrix $\varepsilon(\boldsymbol{x}, \boldsymbol{y})$ "frozen" at a certain value $\boldsymbol{x}=\boldsymbol{x}_{0}$, and then 2) Use the so-called "Bloch measure" technique ( $c f$. [3]) to show that as $\eta \rightarrow 0$, the Bloch components of these approximate eigenfunctions correspond to Bloch eigenvalues concentrating near $\mu$. Hence, we start be proving the following "macroscopic localisation" lemma.

Lemma 3. Let $\mu_{\eta}$ be a sequence of eigenvalues for the problem

$$
\eta^{2} \operatorname{curl}\left(\varepsilon^{-1}(\boldsymbol{x}, \boldsymbol{x} / \eta) \operatorname{curl} \boldsymbol{u}_{\eta}\right)+\boldsymbol{u}_{\eta}=\mu_{\eta}^{-1} \boldsymbol{u}_{\eta}
$$

that converges to a limit $\mu$, and $\boldsymbol{u}_{\eta} \in \mathcal{H}_{\mathrm{per}}(\mathbf{c u r l}, \mathbf{T})$ is the sequence of the corresponding eigenfunctions.

Then there exists a subsequence of $\eta_{j}$, a point $\boldsymbol{x}_{0} \in \mathbf{T}$, and a sequence $\tilde{\boldsymbol{u}}_{j} \in \mathcal{H}_{\text {per }}(\mathbf{c u r l}, \mathbf{T}),\left\|\tilde{\boldsymbol{u}}_{j}\right\|_{\mathbf{L}^{2}(\mathbf{T})}=1$, of quasi-eigenfunctions for the matrices $\varepsilon^{-1}\left(\boldsymbol{x}_{0}, \boldsymbol{x} / \eta_{j}\right)$, i.e. $\mathbf{T}$-periodic solutions of an equation of the form

$$
\eta_{j}^{2} \operatorname{curl}\left(\varepsilon^{-1}\left(\boldsymbol{x}_{0}, \boldsymbol{x} / \eta_{j}\right) \operatorname{curl} \tilde{\boldsymbol{u}}_{j}\right)+\tilde{\boldsymbol{u}}_{j}=\mu_{\eta_{j}}^{-1} \tilde{\boldsymbol{u}}_{j}+\boldsymbol{r}_{j}
$$

where $\boldsymbol{r}_{\eta}$ is a remainder term, which is $\mathbf{T}$-periodic and is such that ${ }^{6}$

$$
\left(\left\|\boldsymbol{w}_{j}\right\|_{\mathbf{L}^{2}(\mathbf{T})}+\left\|\operatorname{curl}_{j}\right\|_{\mathbf{L}^{2}(\mathbf{T})}\right)^{-1}\left\langle\boldsymbol{r}_{j}, \boldsymbol{w}_{j}\right\rangle_{\mathcal{H}^{-1}, \mathcal{H}_{\mathrm{per}}(\operatorname{curl}, \mathbf{T})} \stackrel{\eta \rightarrow 0}{\longrightarrow} 0
$$

for any sequence of non-zero $\boldsymbol{w}_{j} \in \mathcal{H}_{\mathrm{per}}(\mathbf{c u r l}, \mathbf{T})$.

Proof. See the Appendix.

We are now in a position to execute the second step of the plan proposed at the beginning of the section, which we formulate next.

Theorem 2. Let $\boldsymbol{u}_{\eta} \in \mathcal{H}_{\text {per }}(\mathbf{c u r l}, \mathbf{T}), \eta \in \Xi$, be a sequence of eigenfunctions for the problem (4.15), such that $\left\|\boldsymbol{u}_{\eta}\right\|_{\mathbf{L}^{2}(\mathbf{T})}=1$ and $\lim _{\eta \rightarrow 0} \mu_{\eta}=\mu$. Then the limit $\mu$ belongs to the Bloch spectrum $\sigma_{\mathrm{Bloch}}$.

\footnotetext{
${ }^{6}$ We define $\mathcal{H}^{-1}$ as the space of linear bounded functionals on $\mathcal{H}_{\text {per }}(\mathbf{c u r l}, \mathbf{T})$, and $\langle\cdot, \cdot\rangle_{\mathcal{H}^{-1}, \mathcal{H}_{\text {per }}(\mathbf{c u r l}, \mathbf{T})}$ denotes the duality between $\mathcal{H}^{-1}$ and $\mathcal{H}_{\text {per }}($ curl, $\mathbf{T})$.
} 
Proof. Using Lemma 3, select first a subsequence $\eta_{j}$ such that the matrices $\varepsilon^{-1}\left(\boldsymbol{x}_{0}, \boldsymbol{x} / \eta_{j}\right)$ for some $\boldsymbol{x}_{0}$ possess quasi-eigenfunctions $\tilde{\boldsymbol{u}}_{j}$. Consider the respective Bloch-wave decompositions of the functions $\tilde{\boldsymbol{u}}_{j}$ in the torus $\mathbf{T}$ as the union of periodicity cells of size $\eta_{j}$ :

$$
\tilde{\boldsymbol{u}}_{j}(\boldsymbol{x})=\sum_{m} \boldsymbol{u}_{j}^{m}\left(\boldsymbol{x} / \eta_{j}\right) \exp \left(\pi \mathrm{i} T^{-1} \boldsymbol{m} \cdot \boldsymbol{x}\right)
$$

where $\boldsymbol{u}_{j}^{m} \in \mathcal{H}_{\text {per }}(\mathbf{c u r l}, Y)$ for every multi-index $\boldsymbol{m}$ and the summation in (4.17) is performed over all multi-indices $\boldsymbol{m}$ with components $m_{s}, s=1,2,3$, running from 0 to $\eta_{j}^{-1} 2 T-1$.

Further, there is a sequence of (complex) coefficients $\alpha_{j}^{k}(\boldsymbol{m}), k=1,2, \ldots$, such that

$$
\boldsymbol{u}_{j}^{m}(\boldsymbol{y})=\sum_{k \geq 1} \alpha_{j}^{k}(\boldsymbol{m}) \boldsymbol{v}^{k}\left(K_{j}^{-1} \boldsymbol{m}, \boldsymbol{y}\right)
$$

where $K_{j}:=\eta_{j}^{-1} 2 T=2^{2 l_{j}}$, and the functions $\boldsymbol{v}^{k}(\boldsymbol{\theta}, \boldsymbol{y}), k=1,2, \ldots$ are the $\mathbf{L}^{2}(\mathbf{T})$-normalised $\boldsymbol{\theta}$-quasiperiodic eigenfunctions of the operator $\operatorname{curl}_{y} \varepsilon^{-1}\left(\boldsymbol{x}_{0}, \boldsymbol{y}\right) \mathbf{c u r l}_{y}$ defined on $\mathcal{H}_{\text {per }}(\mathbf{c u r l}, Y), c f$. (1.7). Consider a uniformly bounded sequence $\left\{\psi^{k}(\boldsymbol{\theta})\right\}_{k=1}^{\infty}$ of real-valued continuous $Y$-periodic functions, which will be specified at a later stage, and define

$$
\mathcal{M} \tilde{\boldsymbol{u}}_{j}(\boldsymbol{x}):=\sum_{m} \sum_{k \geq 1} \psi^{k}\left(K_{j}^{-1} \boldsymbol{m}\right) \alpha_{j}^{k}(\boldsymbol{m}) \boldsymbol{v}^{k}\left(K_{j}^{-1} \boldsymbol{m}, \boldsymbol{x} / \eta_{j}\right) \exp \left(\pi \mathrm{i} T^{-1} \boldsymbol{m} \cdot \boldsymbol{x}\right) .
$$

Clearly, the function $\mathcal{M} \tilde{\boldsymbol{u}}_{j}$ belongs to $\mathcal{H}_{\text {per }}(\mathbf{c u r l}, \mathbf{T})$ and satisfies the same a priori estimates as $\tilde{\boldsymbol{u}}_{j}$ :

$$
\left\|\mathcal{M} \tilde{\boldsymbol{u}}_{j}\right\|_{\mathbf{L}^{2}(\mathbf{T})}+\eta_{j}\left\|\operatorname{curl} \mathcal{M} \tilde{\boldsymbol{u}}_{j}\right\|_{\mathbf{L}^{2}(\mathbf{T})} \leq C
$$

for some $C>0$. Furthermore, recalling the expression (7.40) for the term $\boldsymbol{r}_{j}$, we have

$$
\begin{gathered}
\left\langle\boldsymbol{r}_{j}, \overline{\mathcal{M} \tilde{\boldsymbol{u}}_{j}}\right\rangle_{\mathcal{H}^{-1}, \mathcal{H}_{\mathrm{per}}(\operatorname{curl}, \mathbf{T})}=\eta_{j}^{2} \int_{\mathbf{T}} \varepsilon^{-1}\left(\boldsymbol{x}_{0}, \boldsymbol{x} / \eta_{j}\right) \operatorname{curl} \tilde{\boldsymbol{u}}_{j} \cdot \operatorname{curl} \overline{\mathcal{M} \tilde{\boldsymbol{u}}_{j}} \mathrm{~d} \boldsymbol{x}+\left(1-\mu_{\eta_{j}}^{-1}\right) \int_{\mathbf{T}} \tilde{\boldsymbol{u}}_{j} \cdot \overline{\mathcal{M} \tilde{\boldsymbol{u}}_{j}} \mathrm{~d} \boldsymbol{x} \\
=(2 T)^{3} \sum_{m} \sum_{k \geq 1} \psi^{k}\left(K_{j}^{-1} \boldsymbol{m}\right)\left|\alpha_{j}^{k}(\boldsymbol{m})\right|^{2}\left(1 / \mu^{k}\left(\boldsymbol{x}_{0}, K_{j}^{-1} \boldsymbol{m}\right)-\mu_{\eta_{j}}^{-1}\right)
\end{gathered}
$$

hence the last expression tends to zero as $j \rightarrow \infty$.

Now define a sequence $\left\{\nu_{j}^{k}\right\}_{k=1}^{\infty}$ of Bloch measures on $Y$, associated with the sequence $\tilde{\boldsymbol{u}}_{j}$ via

$$
\nu_{j}^{k}=(2 T)^{3} \sum_{m}\left|\alpha_{j}^{k}(\boldsymbol{m})\right|^{2} \delta_{\theta=K_{j}^{-1} m}
$$

where $\delta_{\theta=\theta_{0}}$ is the Dirac mass at the point $\boldsymbol{\theta}_{0}$. In view of the fact that the function $\tilde{\boldsymbol{u}}_{j}$ has a unit norm in $\mathbf{L}^{2}(\mathbf{T})$, we get

$$
\sum_{k \geq 1} \int_{Y} \mathrm{~d} \nu_{j}^{k}(\boldsymbol{\theta})=1 \quad \text { for every } j .
$$

In particular, for every $k$, the set $\left\{\nu_{j}^{k}\right\}_{j \geq 1}$ is bounded in the space of Radon measures on $Y$ and hence contains a subsequence that converges weakly ${ }^{7}$ as $j \rightarrow \infty$ to a measure $\nu^{k}$. Note that

$$
\sum_{k \geq 1} \int_{Y} \mathrm{~d} \nu_{j}^{k}(\boldsymbol{\theta})=1
$$

for if we assume the opposite then we should be able to find a constant $\delta>0$, and sequences $j_{l}$ and $k_{l}$ such that

$$
\sum_{k \geq k_{l}} \int_{Y} \mathrm{~d} \nu_{j_{l}}^{k_{l}}(\boldsymbol{\theta}) \geq \delta .
$$

On the other hand, for any $j$,

$$
\eta_{j}^{2}\left\|\operatorname{curl} \tilde{\boldsymbol{u}}_{j}\right\|_{L^{2}(\mathbf{T})^{3}}^{2}=(2 T)^{3} \sum_{m} \sum_{k \geq 1}\left|\alpha_{j}^{k}(\boldsymbol{m})\right|^{2}\left\|\operatorname{curl} \boldsymbol{v}^{k}\left(K_{j}^{-1} \boldsymbol{m}, \boldsymbol{y}\right)+2 \pi \mathrm{i} K_{j}^{-1} \boldsymbol{m} \times \boldsymbol{v}^{k}\left(K_{j}^{-1} \boldsymbol{m}, \boldsymbol{y}\right)\right\|_{\mathbf{L}^{2}(Y)}^{2},
$$

\footnotetext{
${ }^{7}$ More precisely, "weakly*" if the space of Radon measures is considered as the dual of $C_{\text {per }}(Y)$.
} 
and since in view of the equation defining the functions $\boldsymbol{v}^{k}(\boldsymbol{\theta}, \boldsymbol{y})$ one has

$$
\left\|\operatorname{curl} \boldsymbol{v}^{k}(\boldsymbol{\theta}, \boldsymbol{y})+2 \pi \mathrm{i} \boldsymbol{\theta} \times \boldsymbol{v}^{k}(\boldsymbol{\theta}, \boldsymbol{y})\right\|_{\mathbf{L}^{2}(Y)}^{2}=1 / \mu^{k}\left(\boldsymbol{x}_{0}, \boldsymbol{\theta}\right)-1,
$$

for any $k, \boldsymbol{\theta}$, we deduce

$$
\eta_{j}^{2}\left\|\operatorname{curl} \tilde{\boldsymbol{u}}_{j}\right\|_{\mathbf{L}^{2}(\mathbf{T})} \geq \delta \min _{\theta \in Y}\left(1 / \mu^{k_{l}}\left(\boldsymbol{x}_{0}, \boldsymbol{\theta}\right)-1\right)
$$

which goes to $+\infty$ as $l \rightarrow \infty$ since for any $\boldsymbol{\theta} \in Y$, we have $\lim _{k \rightarrow \infty} \mu^{k}\left(\boldsymbol{x}_{0}, \boldsymbol{\theta}\right)=0$, hence contradiction with the fact that $\eta_{j} \operatorname{curl} \tilde{\boldsymbol{u}}_{j}$ is bounded in $\mathbf{L}^{2}(\mathbf{T})$.

As we noted above (see (4.18) and the text that follows),

$$
\sum_{k \geq 1} \int_{Y} \psi^{k}(\boldsymbol{\theta})\left(1 / \mu^{k}\left(\boldsymbol{x}_{0}, \boldsymbol{\theta}\right)-1 / \mu_{\eta_{j}}\right) \mathrm{d} \nu_{j}^{k}(\boldsymbol{\theta}) \rightarrow 0 \quad \text { as } \quad j \rightarrow \infty
$$

which, having passed to the limit, can be re-written as

$$
\sum_{k \geq 1} \int_{Y} \psi^{k}(\boldsymbol{\theta})\left(1 / \mu^{k}\left(\boldsymbol{x}_{0}, \boldsymbol{\theta}\right)-1 / \mu\right) \mathrm{d} \nu^{k}(\boldsymbol{\theta})=0
$$

Since in view of (4.19) at least one of the measures $\nu^{k}$ is non-zero, by choosing appropriately the sequence $\psi^{k}(\boldsymbol{\theta})$ we can ensure that (4.20) implies the existence of $k$ and $\boldsymbol{\theta}$ such that $\mu=\mu^{k}\left(\boldsymbol{x}_{0}, \boldsymbol{\theta}\right)$.

Corollary 2. For any sequence of $\eta \in \Xi$, one has $\lim _{\eta \rightarrow 0} \eta^{-2} \sigma_{\eta}=\left\{(1-\Lambda)^{-1} \Lambda: \Lambda \in \sigma_{\text {Bloch }}\right\}$.

\section{5 "Non-critical" scalings}

In this section we study pairs $\left(\mu_{\eta}, \boldsymbol{H}_{\eta}\right) \in \mathbb{R}^{+} \times \mathcal{H}_{\text {per }}(\mathbf{c u r l}, \mathbf{T}), \boldsymbol{H}_{\eta} \not \equiv \mathbf{0}$, such that

$$
a_{\eta}^{2} \operatorname{curl}\left(\varepsilon^{-1}(\boldsymbol{x}, \boldsymbol{x} / \eta) \operatorname{curl} \boldsymbol{H}_{\eta}\right)+\boldsymbol{H}_{\eta}=\mu_{\eta}^{-1} \boldsymbol{H}_{\eta}
$$

where $a_{\eta} \rightarrow 0$. The equation (5.21) is obtained from (1.1) by setting $\mu_{\eta}^{k}=\left(a_{\eta}^{2}+\Lambda_{\eta}^{k}\right)^{-1} \Lambda_{\eta}^{k}$ and keeping the corresponding eigenfunctions unchanged. Analogously to what was done in Section 4, we introduce an operator $\mathcal{S}_{\eta} \in \mathcal{L}\left(\mathbf{L}^{2}(\mathbf{T})\right)$ defined for every $\boldsymbol{f} \in \mathbf{L}^{2}(\mathbf{T})$ by $\mathcal{S}_{\eta} \boldsymbol{f}=\boldsymbol{v}_{\eta}$, where $\boldsymbol{v}_{\eta}$ is the unique solution in $\mathcal{H}_{\text {per }}($ curl, $\mathbf{T})$ of

$$
a_{\eta}^{2} \operatorname{curl}\left(\varepsilon^{-1}(\boldsymbol{x}, \boldsymbol{x} / \eta) \operatorname{curl} \boldsymbol{v}_{\eta}\right)+\boldsymbol{v}_{\eta}=\boldsymbol{f}
$$

The analysis of the convergence of the sequence of operators $\mathcal{S}_{\eta}$ can be carried out in the same way as before. However, outside the scaling regime $a_{\eta} \sim \eta$ there is no interaction between the homogenisation scale $\eta$ and the singular perturbation scale $a_{\eta}$. One of two things can happen instead: either $\eta$ is much smaller than $a_{\eta}$ in which case the homogenisation occurs first and the singular perturbation concerns the homogenised system; or $\eta$ is larger than $a_{\eta}$ and it turns out that the singular perturbation occurs first at a microscopic scale making the homogenisation irrelevant. An appropriate tool to tackle these two cases is the notion of multi-scale convergence, introduced in [5]. We recall the main results of [5] and adapt them, where appropriate, to the three-scale convergence of function and operator sequences.

\subsection{Large scales: $1 \gg a_{\eta} \gg \eta$}

Definition 2. Consider a bounded set $\mathbf{T} \subset \mathbb{R}^{N}$ and $Z:=[0, l]^{N}$, where $l \in \mathbb{N}$. Let $a_{\eta}$ be a sequence of positive numbers converging to zero, $\eta \in \Xi$.

i) A function $\boldsymbol{\varphi} \in \mathbf{L}^{2}(\mathbf{T} \times Z \times Y)$ is said to be (three-scale) admissible if the sequence $\boldsymbol{\varphi}_{\eta}(\boldsymbol{x}):=$ $\boldsymbol{\varphi}\left(\boldsymbol{x}, l \boldsymbol{x} / a_{\eta}, \boldsymbol{x} / \eta\right)$ is bounded in $\mathbf{L}^{2}(\mathbf{T})$ and satisfies

$$
\lim _{\eta \rightarrow 0} \int_{\mathbf{T}}\left|\boldsymbol{\varphi}_{\eta}(\boldsymbol{x})\right|^{2} \mathrm{~d} \boldsymbol{x}=\frac{1}{l^{N}} \int_{\mathbf{T}} \int_{Z} \int_{Y}|\boldsymbol{\varphi}(\boldsymbol{x}, \boldsymbol{z}, \boldsymbol{y})| \mathrm{d} \boldsymbol{y} \mathrm{d} \boldsymbol{z} \mathrm{d} \boldsymbol{x} .
$$

ii) A sequence $\boldsymbol{u}_{\eta}$ of functions in $\mathbf{L}^{2}(\mathbf{T})$ is said to weakly three-scale converge to $\boldsymbol{u}_{0}(\boldsymbol{x}, \boldsymbol{z}, \boldsymbol{y}) \in \mathbf{L}^{2}(\mathbf{T} \times Z \times$ $Y)$, and in this case we write $\boldsymbol{u}_{\eta}-\boldsymbol{u}_{0}$, if for any admissible $\boldsymbol{\psi} \in L^{2}(\mathbf{T} \times Z \times Y)$, e.g. $\boldsymbol{\psi} \in C\left(\mathbf{T}, \mathbf{C}_{\text {per }}^{\infty}(Z \times Y)\right)$, one has

$$
\lim _{\eta \rightarrow 0} \int_{\mathbf{T}} \boldsymbol{u}_{\eta}(\boldsymbol{x}) \cdot \boldsymbol{\psi}\left(\boldsymbol{x}, l \boldsymbol{x} / a_{\eta}, \boldsymbol{x} / \eta\right) \mathrm{d} \boldsymbol{x}=\frac{1}{l^{N}} \int_{\mathbf{T}} \int_{Z} \int_{Y} \boldsymbol{u}_{0}(\boldsymbol{x}, \boldsymbol{z}, \boldsymbol{y}) \cdot \boldsymbol{\psi}(\boldsymbol{x}, \boldsymbol{z}, \boldsymbol{y}) \mathrm{d} \boldsymbol{y} \mathrm{d} \boldsymbol{z} \mathrm{d} \boldsymbol{x} .
$$


Theorem 3. Consider $\mathbf{T}:=[-T, T]^{3}, T>0$, and $\eta \in \Xi$. For a sequence of positive numbers $a_{\eta}, \eta \in \Xi$, such that $\lim _{\eta \rightarrow 0} a_{\eta}=0$ and $\lim _{\eta \rightarrow 0} \eta^{-1} a_{\eta}=\infty$, we look for $\boldsymbol{u}_{\eta} \in \mathcal{H}_{\mathrm{per}}(\mathbf{c u r l}, \mathbf{T})$ that are weak solutions of

$$
a_{\eta}^{2} \operatorname{curl}\left(\varepsilon^{-1}(\boldsymbol{x}, \boldsymbol{x} / \eta) \operatorname{curl} \boldsymbol{u}_{\eta}\right)+\boldsymbol{u}_{\eta}=\boldsymbol{f}_{\eta}
$$

with $\varepsilon(\boldsymbol{x}, \boldsymbol{y}) \in C_{\mathrm{per}}\left(\mathbf{T},\left[L^{\infty}(Y)\right]^{9}\right)$ coercive and $\boldsymbol{f}_{\eta} \in \mathbf{L}^{2}(\mathbf{T})$.

i) The equation (5.22) has a unique solution $\boldsymbol{u}_{\eta} \in \mathcal{H}_{\text {per }}(\mathbf{c u r l}, \mathbf{T})$.

ii) Let $l \in \mathbb{N}$ and suppose that $\boldsymbol{f}_{\eta} \rightarrow \boldsymbol{f} \in \mathbf{L}^{2}(\mathbf{T} \times Z)$. There is a subsequence of problems (5.22), for which we keep the same index $\eta$, such that $\boldsymbol{u}_{\eta}-\boldsymbol{u}_{0}+\boldsymbol{u}_{1}$ and $a_{\eta} \mathbf{c u r l}_{\eta}-\boldsymbol{c u r l}_{z} \boldsymbol{u}_{1}+\boldsymbol{u}_{2}$, where $\boldsymbol{u}_{0} \in L^{2}\left(\mathbf{T} \times Z, \mathbf{L}_{\text {pot }}^{2}(Y)\right), \boldsymbol{u}_{1} \in L^{2}\left(\mathbf{T}, \mathcal{H}_{\text {per }}(\operatorname{curl}, Z)\right), \boldsymbol{u}_{2} \in L^{2}\left(\mathbf{T} \times Z, \mathbf{L}_{\text {sol }}^{2}(Y)\right)$, and for almost every $\boldsymbol{x} \in \mathbf{T}$ the function $\boldsymbol{u}_{1}(\boldsymbol{x}, \cdot)$ is the unique solution of

$$
\operatorname{curl}_{z}\left(\varepsilon_{\mathrm{hom}}^{-1}(\boldsymbol{x}) \operatorname{curl}_{z} \boldsymbol{u}_{1}(\boldsymbol{x}, \boldsymbol{z})\right)+\boldsymbol{u}_{1}(\boldsymbol{x}, \boldsymbol{z})=\boldsymbol{f}(\boldsymbol{x}, \boldsymbol{z}) .
$$

where $\varepsilon_{\mathrm{hom}}(\boldsymbol{x})$ is given by the formula (1.5) (or, equivalently, (1.4)).

iii) Let $\boldsymbol{u}_{\eta}$ be the three-scale convergent subsequence from ii). Then $\boldsymbol{u}_{\eta} \rightarrow \boldsymbol{u}_{1}$, hence the operator sequence $\mathcal{S}_{\eta}$ defined in $\mathbf{L}^{2}(\mathbf{T})$ by $\mathcal{S}_{\eta} \boldsymbol{f}_{\eta}=\boldsymbol{u}_{\eta}$ strongly two-scale converges to $\mathcal{S}^{l}$ defined in $\mathbf{L}^{2}(\mathbf{T} \times Z)$ by the formula $\mathcal{S}^{l} \boldsymbol{f}=\boldsymbol{u}_{1}$, where $\boldsymbol{u}_{1} \in L^{2}\left(\mathbf{T}, \mathcal{H}_{\text {per }}(\mathbf{c u r l}, Z)\right)$ is the unique solution of (5.23).

Proof. See the Appendix.

Corollary 3. For any sequence $a_{\eta}, \eta \in \Xi$, as in Theorem 3,

$$
\lim _{\eta \rightarrow 0} \sigma\left(\mathcal{S}_{\eta}\right)=[0,1]
$$

where the limit is understood in the Hausdorff sense (see the footnote on p. 3). Equivalently, the limit of $a_{\eta}^{-2} \sigma_{\eta}$ is the entire positive real axis.

Proof. From Lemma 2, we deduce that for a relevant subsequence,

$$
\sigma\left(\mathcal{S}^{l}\right) \subset \lim _{\eta \rightarrow 0} \sigma\left(\mathcal{S}_{\eta}\right)
$$

Moreover, for any $l \in \mathbb{N}$, the spectrum $\sigma\left(\mathcal{S}^{l}\right)$ is obtained from $\sigma\left(\mathcal{S}^{1}\right)$ by a simple transformation since the coefficient matrix in (5.23) does not depend on $\boldsymbol{z}$. Labelling in increasing order the eigenvalues $\gamma_{l}^{k}, k \geq 1$, of $S^{l}$, they satisfy the identity $\gamma_{l}^{k}=\left(l^{2} \gamma_{1}^{k}+\left(1-\gamma_{1}^{k}\right)\right)^{-1} l^{2} \gamma_{1}^{k}$. Using this and the fact that $\gamma_{1}^{k} \rightarrow 0$ as $k \rightarrow \infty$, it can be shown that the set $\left\{\gamma_{l}^{k}, k, l \in \mathbb{N}\right\} \equiv \bigcup_{l \geq 1} \sigma\left(\mathcal{S}^{l}\right)$ is dense in $[0,1]$. In view of (5.25), via a simple argument, this implies (5.24).

Remark 2. Given a sequence $a_{\eta}, \eta \in \Xi$, the statement of Corollary 3 clearly also holds for any subsequence of $a_{\eta}, \eta \in \Xi$.

\subsection{Small scales: $a_{\eta} \ll \eta$}

Definition 3. Consider a bounded set $\mathbf{T} \subset \mathbb{R}^{N}$ and $Z:=[0, l]^{N}$, where $l \in \mathbb{N}$. Let $a_{\eta}, \eta \in \Xi$, be a sequence of positive numbers such that $\lim _{\eta \rightarrow 0} a_{\eta}=0$.

i) Let $\boldsymbol{u}_{\eta}$ be a sequence of functions in $\mathbf{L}^{2}(\mathbf{T})$ that weakly three-scale converges to $\boldsymbol{u}_{0}(\boldsymbol{x}, \boldsymbol{z}, \boldsymbol{y}) \in \mathbf{L}^{2}(\mathbf{T} \times$ $Z \times Y)$. Suppose that

$$
\lim _{\eta \rightarrow 0} \int_{\mathbf{T}}\left|\boldsymbol{u}_{\eta}(\boldsymbol{x})\right|^{2} \mathrm{~d} \boldsymbol{x}=\frac{1}{l^{N}} \int_{\mathbf{T}} \int_{Z} \int_{Y}\left|\boldsymbol{u}_{0}(\boldsymbol{x}, \boldsymbol{z}, \boldsymbol{y})\right|^{2} \mathrm{~d} \boldsymbol{y} \mathrm{d} \boldsymbol{z} \mathrm{d} \boldsymbol{x} .
$$

Then $\boldsymbol{u}_{\eta}$ is said to strongly three-scale converge to $\boldsymbol{u}_{0}(\boldsymbol{x}, \boldsymbol{z}, \boldsymbol{y})$, and we write $\boldsymbol{u}_{\eta} \rightarrow \boldsymbol{u}_{0}$.

ii) A sequence $\mathcal{S}_{\eta}$ of operators in $\mathbf{L}^{2}(\mathbf{T})$ is said to strongly three-scale converge to the operator $\mathcal{S}$ in $\left.\mathbf{L}^{2}(\mathbf{T} \times Z \times Y)\right)$ if for any sequence $f_{\eta} \in \mathbf{L}^{2}(\mathbf{T})$ such that $\boldsymbol{f}_{\eta} \rightarrow \boldsymbol{f}$ one has $\mathcal{S}_{\eta} \boldsymbol{f}_{\eta} \rightarrow \mathcal{S} \boldsymbol{f}$.

Theorem 4. Suppose that the sequence $a_{\eta}, \eta \in \Xi$ is such that $\lim _{\eta \rightarrow 0} \eta^{-1} a_{\eta}=0$. Consider $\mathbf{T}:=[-T, T]^{3}$, $T>0$, and for any $\eta \in \Xi$ look for $\boldsymbol{u}_{\eta} \in \mathcal{H}_{\mathrm{per}}(\mathbf{c u r l}, \mathbf{T})$ satisfying (in the weak sense)

$$
a_{\eta}^{2} \operatorname{curl}\left(\varepsilon^{-1}(\boldsymbol{x}, \boldsymbol{x} / \eta) \operatorname{curl} \boldsymbol{u}_{\eta}\right)+\boldsymbol{u}_{\eta}=\boldsymbol{f}_{\eta}
$$

with $\varepsilon(\boldsymbol{x}, \boldsymbol{y}) \in C_{\mathrm{per}}\left(\mathbf{T},\left[L^{\infty}(Y)\right]^{9}\right)$ coercive and $\boldsymbol{f}_{\eta} \in \mathbf{L}^{2}(\mathbf{T})$.

i) The equation (5.26) has a unique solution $\boldsymbol{u}_{\eta} \in \mathcal{H}_{\mathrm{per}}(\mathbf{c u r l}, \mathbf{T})$.

\footnotetext{
${ }^{8}$ The space $\mathbf{L}_{\text {pot }}^{2}(Y)$ is defined as the closure of the set $\left\{\nabla \varphi: \varphi \in C_{\text {per }}^{\infty}(Y)\right\}$ in the norm of $\mathbf{L}^{2}(Y)$.
} 
ii) Suppose that $\boldsymbol{f}_{\eta} \rightarrow \boldsymbol{f} \in \mathbf{L}^{2}(\mathbf{T} \times Z \times Y)$. For any $l \in \mathbb{N}$ there is a subsequence of problems (5.26), for which we keep the same index $\eta$, such that $\boldsymbol{u}_{\eta}-\boldsymbol{u}_{0}$ and $a_{\eta} \boldsymbol{c u r l}_{\eta}-\boldsymbol{c u r l}_{z} \boldsymbol{u}_{0}$, where $\boldsymbol{u}_{0} \in \mathbf{L}^{2}(\mathbf{T} \times$ $\left.Y, \mathcal{H}_{\mathrm{per}}(\mathbf{c u r l}, Z)\right)$ and for almost every $\boldsymbol{x} \in \mathbf{T}, \boldsymbol{y} \in Y$, the function $\boldsymbol{u}_{0}(\boldsymbol{x}, \cdot, \boldsymbol{y})$ is the unique solution of

$$
\operatorname{curl}_{z}\left(\varepsilon^{-1}(\boldsymbol{x}, \boldsymbol{y}) \operatorname{curl}_{z} \boldsymbol{u}_{0}\right)+\boldsymbol{u}_{0}=\boldsymbol{f}(\boldsymbol{x}, \cdot, \boldsymbol{y}) .
$$

iii) The operator sequence $\mathcal{S}_{\eta}$ defined in $\mathbf{L}^{2}(\mathbf{T})$ by $\mathcal{S}_{\eta} \boldsymbol{f}_{\eta}=\boldsymbol{u}_{\eta}$ strongly three-scale converges to $\mathcal{S}^{l}$ defined in $\mathbf{L}^{2}(\mathbf{T} \times Z)$ by the formula $\mathcal{S}^{l} \boldsymbol{f}=\boldsymbol{u}_{0}$, where $\boldsymbol{u}_{0}$ is the solution of (5.27).

The proof of the above theorem goes along the lines of Theorem 1 and Theorem 3 with some simplifications, and we will not dwell on it here. In the same way as before, a statement on the convergence of the spectra follows.

Corollary 4. For any sequence $a_{\eta}, \eta \in \Xi$, as in Theorem $4, \lim _{\eta \rightarrow 0} \sigma\left(\mathcal{S}_{\eta}\right)=[0,1]$, or, equivalently, the limit of $a_{\eta}^{-2} \sigma_{\eta}$ is the entire positive real axis. The same is true for any subsequence of $a_{\eta}, \eta \in \Xi$.

\section{Numerical example}

In this section we illustrate our spectral convergence result in the critical case $a_{\eta} \sim \eta$ (see Sections 2-4) by considering the propagation of TM-polarised electromagnetic waves through a periodic array of parallel infinitely long cylinders. The corresponding analogue of the problem (1.1) in this case is

$$
-\Delta E(\boldsymbol{x})=\Lambda_{\eta}^{-1} \varepsilon(\boldsymbol{x} / \eta) E(\boldsymbol{x}),
$$

where $\boldsymbol{x} \in[-1,1]^{2}$, and $E$ is the only non-zero component of the electric field, which is parallel to the cylinders. For simplicity, in (6.28) we drop the dependence of the permittivity matrix on the "slow" variable $\boldsymbol{x}$, assume that the medium is isotropic at every point so that $\varepsilon(\boldsymbol{y})$ is a scalar for any $\boldsymbol{y} \in Y$, and set $T=1$.

In our calculations, we set the relative permittivity $\varepsilon=12$ inside and $\varepsilon=1$ outside the cylinders, which corresponds to the situation of straight fibres of a semi-conductor surrounded by air or, more generally, any two materials with a permittivity contrast of 12 (the permittivity of the majority of dielectric materials found in nature ranges from 1 to 14). Here we consider fibres with square cross-section to preserve the four-fold symmetry in the unit cell, hence it is sufficient to consider only a quarter of the Brillouin zone $Y^{*}=[0,2 \pi]^{2}$ ("first reduced Brillouin zone").

The Bloch spectrum of (6.28) is obtained by studying a family of spectral problems on $Y$ associated with (6.28). For any $\boldsymbol{\kappa} \in Y^{*}$ consider pairs $(\Lambda(\boldsymbol{\kappa}), E(\boldsymbol{y})) \in \mathbb{R}^{+} \times H_{\text {per }}^{1}(\mathbf{Y})$ such that $(c f .(1.7))$

$$
-\Delta\left(E(\boldsymbol{y}) e^{i \boldsymbol{\kappa} \cdot \boldsymbol{y}}\right)=\Lambda(\boldsymbol{\kappa})^{-1} \varepsilon(\boldsymbol{y}) E(\boldsymbol{y}) e^{i \boldsymbol{\kappa} \cdot \boldsymbol{y}} .
$$

For every $\boldsymbol{\kappa} \in[0,2 \pi]^{2}$ the related eigenvalues $\Lambda(\boldsymbol{\kappa})$ form a sequence, which we denote by $\left\{\Lambda^{k}(\boldsymbol{\kappa})\right\}_{k \geq 1}$. The frequencies $\Lambda^{k}(\boldsymbol{\kappa})^{-1 / 2}, k=1, \ldots, 10$, as functions of $\boldsymbol{\kappa} \in Y^{*}$ are shown by the surface dispersion diagram in Figure 1. As it may be difficult to visualise these plots, it is customary to restrict oneself to "boundary" dispersion curves, which correspond to $\boldsymbol{\kappa} \in \partial Y^{*}$, assuming

$$
\left[\min _{\kappa \in Y^{*}} \Lambda^{k}(\boldsymbol{\kappa}), \max _{\kappa \in Y^{*}} \Lambda^{k}(\boldsymbol{\kappa})\right]=\left[\min _{\kappa \in \partial Y^{*}} \Lambda^{k}(\boldsymbol{\kappa}), \max _{\kappa \in \partial Y^{*}} \Lambda^{k}(\boldsymbol{\kappa})\right] .
$$

The validity of this assumption appears to be an open problem, but since it helps getting a better idea of the stop band properties, in Figure 2 we plot such dispersion curves corresponding to Figure 1 . We emphasise that we first numerically checked that all of the surfaces shown satisfy the conjecture (6.29). We feel that such a preliminary test on dispersion surfaces should be systematically performed before using the rule (6.29).

We would now like to look at the spectrum associated with the sequence of problems (6.28), as $\eta$ goes to zero. Consider the corresponding differential operator with periodic boundary conditions on the cell $[-1,1]^{2}$ and look for the convergence of its spectrum when the number of inclusions increases and their size tends to zero so that their overall surface remains constant. It is apparent from Figures 3 to 6 that the spectrum becomes denser as $\eta$ goes to zero. It is worth noting that the location of the band gaps can be determined with good accuracy as early as the second step of the Bloch wave homogenisation process i.e. when $\eta=1 / 4$. The mechanism leading to the appearance of the stop bands involves a relaxation of the constraint on the periodic condition: when the number of inclusions increases, the wave satisfies both a periodic condition on the sides of the cell and some phase shift between inclusions. The larger the number of inclusions, the more prominent the phase shift between the inclusions. The consequence is that it is enough to build a large super-cell with periodic boundary conditions to filter Bloch waves within a periodic array. This observation might well prove useful for numerical purposes when implementation of Floquet-Bloch conditions is not straightforward within a package. 


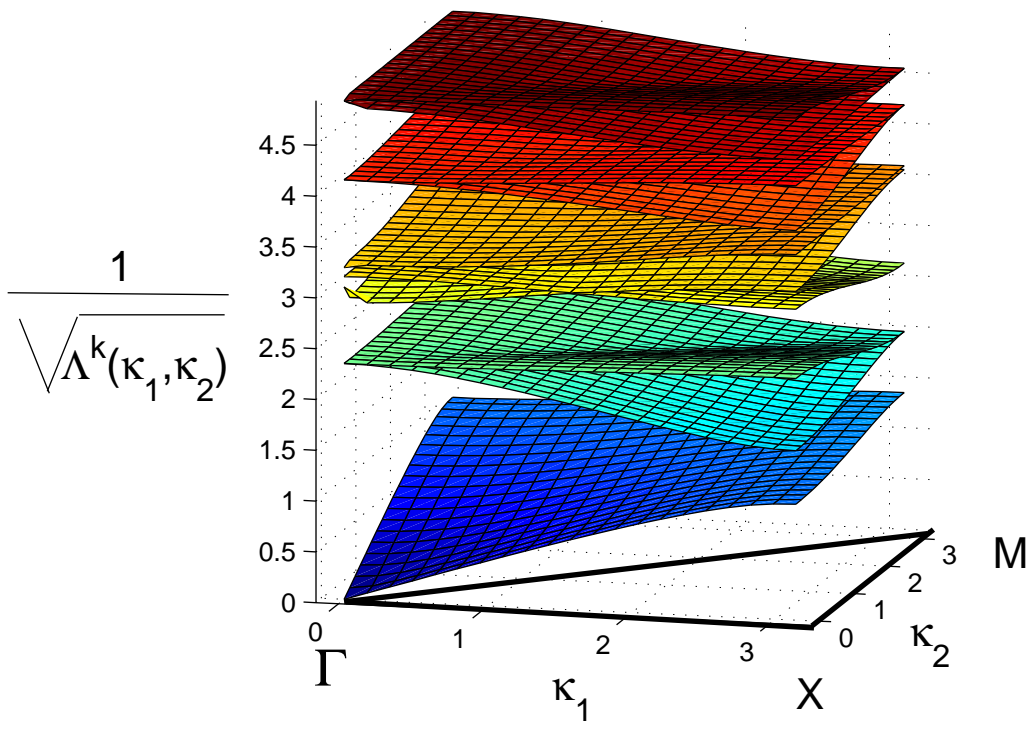

Figure 1: Surface dispersion diagram for TM-waves propagating within a periodic array of square cylinders of a semiconductor $(\varepsilon=12)$ surrounded by air $(\varepsilon=1)$; the cross section of the cylinders is 0.6 and the structure period is 1 . On the vertical axis we show the frequency $1 / \sqrt{\Lambda_{\eta}(\boldsymbol{\kappa})}$, while the horizontal axes correspond to the components of the Bloch vector $\boldsymbol{\kappa}$, which spans the area of the reduced Brillouin zone, i.e. the right-angle triangle with vertices $\Gamma=(0,0), X=(\pi, 0)$ and $M=(\pi, \pi)$.

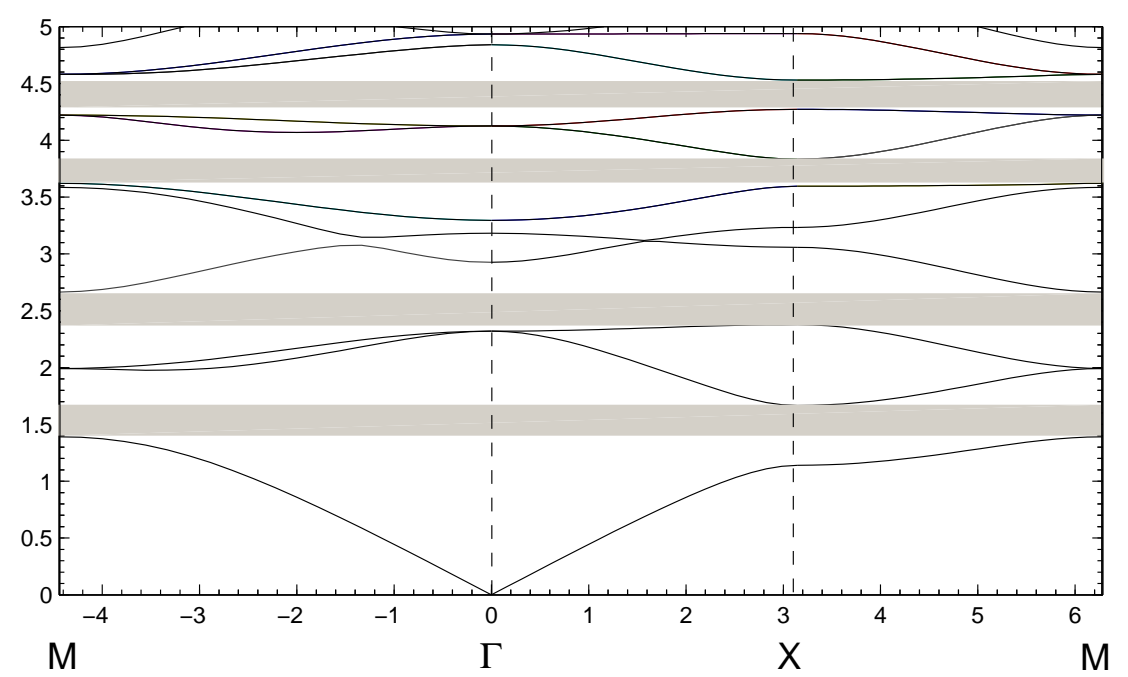

Figure 2: Dispersion curves for TM-modes of Figure 1 when the Bloch vector $\kappa$ runs along the edges of the reduced Brillouin zone $\Gamma M X$. The horizontal axis represents the value of $\kappa_{1}$ from $\Gamma$ to $X$, the value of $\kappa_{1}+\kappa_{2}=\pi+\kappa_{2}$ from $X$ to $M$ and the value of $-\sqrt{\kappa_{1}^{2}+\kappa_{2}^{2}}$ from $M$ to $\Gamma$. Note the presence of four stop bands (shaded regions) for normalised frequencies $1 / \sqrt{\Lambda^{k}(\boldsymbol{\kappa})}$ within the intervals $[1.39,1.67],[2.38,2.66]$, $[3.62,3.83],[4.27,4.53]$.

\section{Discussion}

We have studied the high-frequency asymptotic behaviour of the spectra $\sigma_{\eta}$ of operators $\operatorname{curl} \varepsilon^{-1}(\boldsymbol{x}, \boldsymbol{x} / \eta) \operatorname{curl}$ as $\eta \rightarrow 0$ under the standard ellipticity conditions on the periodic permittivity matrix $\varepsilon^{-1}(\boldsymbol{x}, \boldsymbol{y})$. We have shown that the sets $a_{\eta}^{-2} \sigma_{\eta}$ converge in the Hausdorff sense either to the set $[0,+\infty)$ or to the (Bloch) 


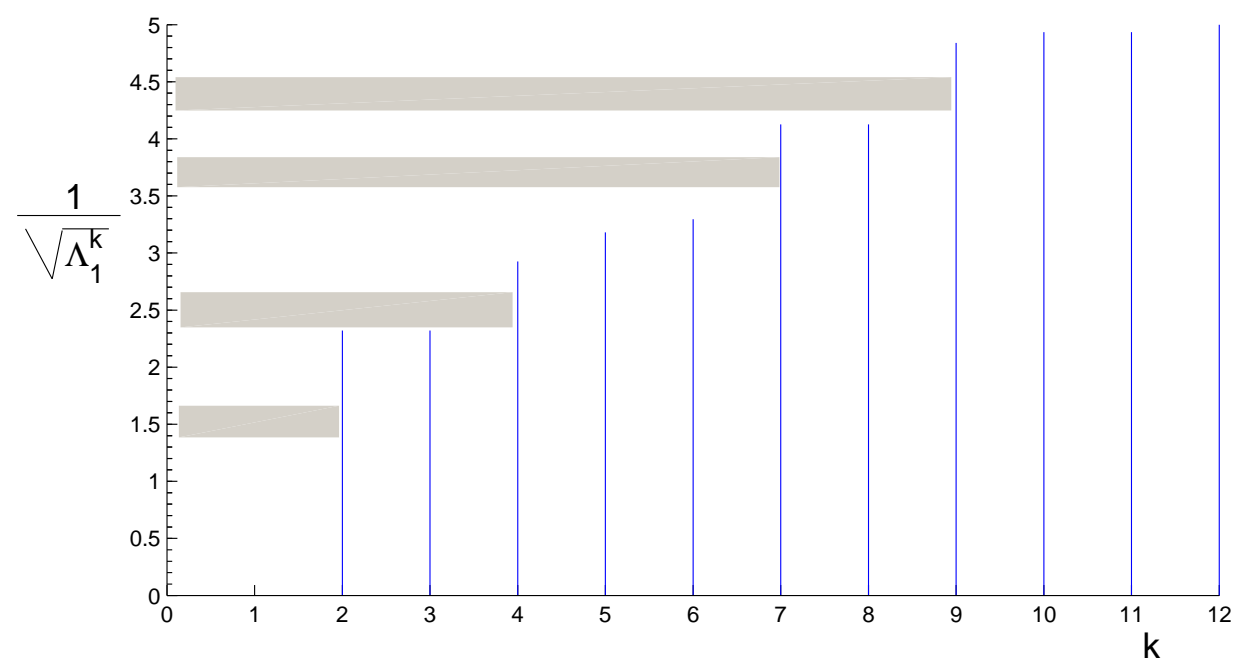

Figure 3: Inverse square root of the first 12 eigenvalues in the discrete spectrum associated with the spectral problem (6.28) on the unit cell $[-1,1]^{2}$ with periodic boundary conditions. In this case the cell contains one square inclusion with side length 0.6. The isolated eigenvalues sit well outside the band gaps of Figure 2, given by shaded regions.

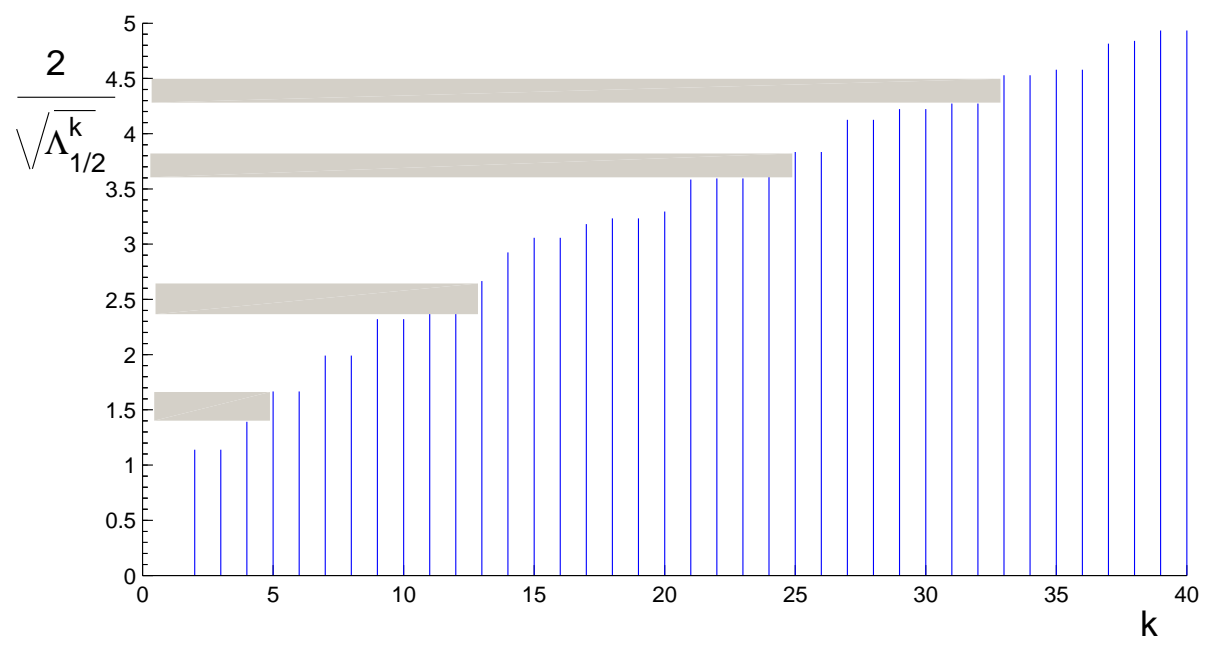

Figure 4: The same setting as in Figure 3, but the cell contains four inclusions with side length $0.3(\eta=1 / 2)$. Here we plot the first 40 rescaled eigenvalues $\Lambda_{1 / 2}^{k}$. The spectrum is denser compared to Figure 3. Notably, some of the eigenvalues are already located on the edge of the band gaps. The location of the stop bands is well determined already for $\eta=1 / 2$. Nevertheless, the eigenvalues remain sparsely located between the gaps.

spectrum of the operator $\operatorname{curl}_{y} \varepsilon^{-1}(\boldsymbol{x}, \boldsymbol{y}) \mathbf{c u r l}_{y}$. The proof of this fact consists of two parts: 1) We introduce the notion of two-scale operator convergence, which, having been applied to the problem in question, implies that rescaled sequences of eigenvalues converge to points in the spectrum of an operator that is "limiting" in some sense; 2) In the case when $a_{\eta} \sim \eta$, a technique based on the construction of "quasi-eigenfunctions" and the associated "Bloch measures" allows us to show that any point in the Bloch spectrum can be attained as the limit of a sequence from 1).

There are several possible directions in which our results could be developed. First, the issue of whether non-periodic boundary conditions could be treated in a similar way, which is of special importance in the electromagnetic context. A crude analysis shows that, although Dirichlet-type conditions could be studied in the same way as in [3], the Neumann-type boundary presents a significant challenge in the description of what is called the "boundary-layer" spectrum. Secondly, the high-frequency spectrum analysis can be considered in the context of high-contrast periodic composites, which has received considerable attention recently in the finite-frequency regime, see [17], [6]. Depending on the exact relationship between the contrast parameter 


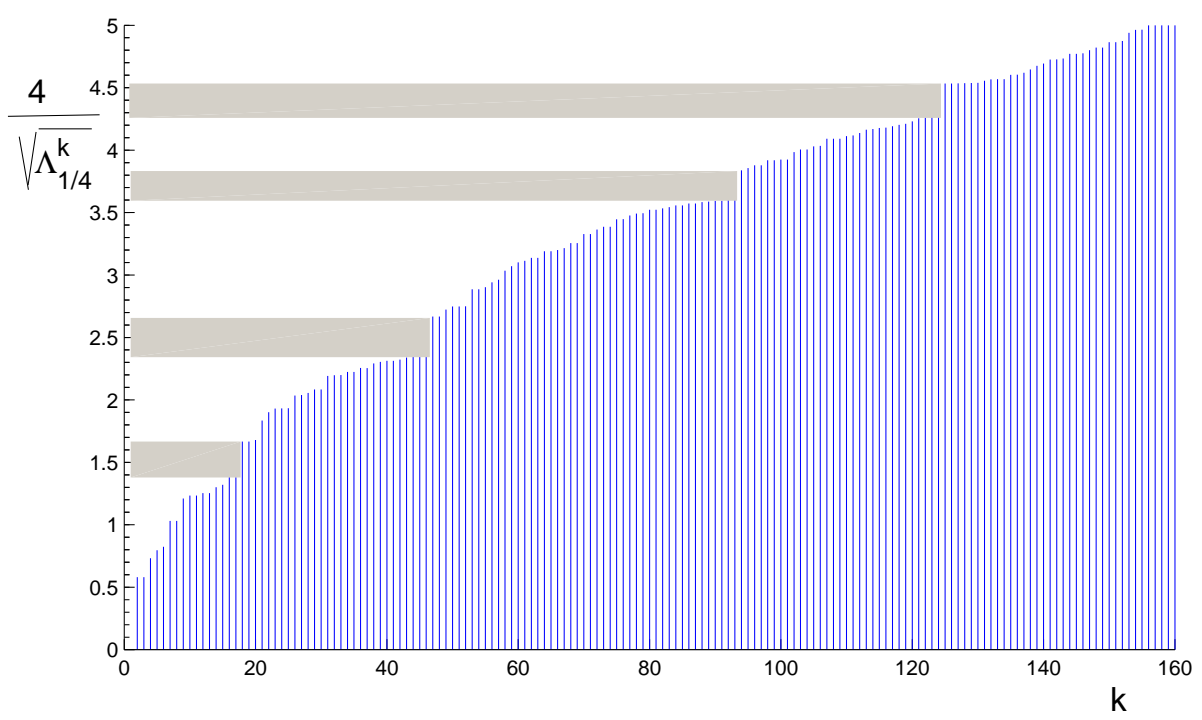

Figure 5: The same setting as in Figure 4 but the cell contains 16 inclusions with side length $0.015(\eta=1 / 4)$. Here we plot the first 160 rescaled eigenvalues $\Lambda_{1 / 4}^{k}$. The four band gaps are now clearly standing out in the dense set of eigenvalues. Comparing Figure 4 and Figure 5 we note that the eigenvalues on the edges of the gaps start degenerating (multiplicity greater than one). One can follow some of the eigenvalues in the limiting process, for instance $\Lambda_{1 / 4}^{15}=\Lambda_{1 / 4}^{16}=16 \Lambda_{1 / 2}^{3} \sim 1.38$.

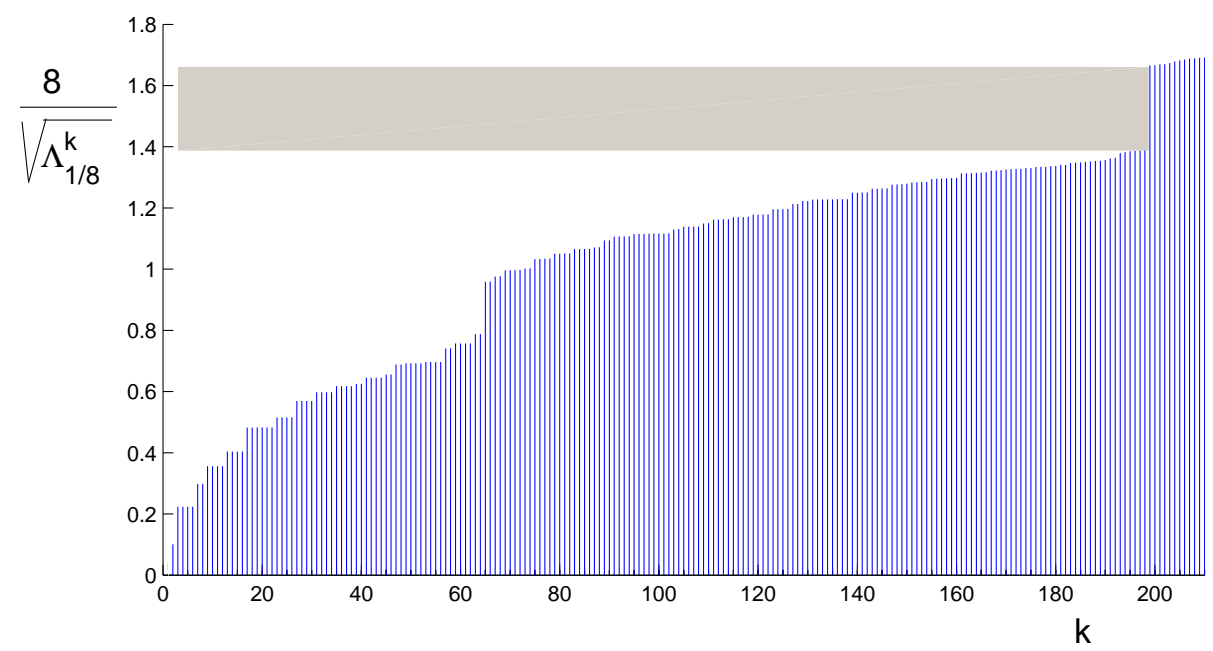

Figure 6: The same setting as in Figure 5 but there are 196 inclusions with side length 0.0075 in the cell $(\eta=1 / 16)$. Here the first 210 eigenvalues $\Lambda_{1 / 16}^{k}$ are shown. We only plot eigenvalues located within the range $[0,1.8]$ for clarity (one should plot over 500 eigenvalues to cover the range $[0,5]$ ).

$\delta$, the period of oscillations $\eta$ and the wavelength $a_{\delta, \eta}$, the limiting problem could possess some or other type of spectrum, possibly leading to a number of new effects in the limit of vanishing parameter values. The band-gap features of the "high-contrast high-frequency" case can then be exploited in creating localised modes in the spectrum by adding a compact perturbation to the limiting operator. This will translate in a suitably rescaled perturbation of the original composite with finite $\delta, \eta$, which possesses the mentioned localisation properties in a controlled way as $\delta$ and $\eta$ vary. These aspects of the high-frequency spectral asymptotics for periodic media will be studied elsewhere. 


\section{Acknowledgements}

KDC would like to acknowledge the EPSRC financial support, on the project EP/C52392X/1. SG was funded by Centre National de la Recherche Scientifique (CNRS), and he also received support from the EU-funded project PHOREMOST under Grant FP6/2003/IST/2-511616.

\section{Appendix: proofs of some of the main statements}

\section{Proof of Theorem 1}

i) The existence and uniqueness of a solution $\boldsymbol{u}_{\eta} \in \mathcal{H}_{\text {per }}(\mathbf{c u r l}, \mathbf{T})$ to the problem (3.13) follows from the standard argument invoking the Lax-Milgram lemma.

ii) The sequences $\boldsymbol{u}_{\eta}$ and $\eta \mathbf{c u r l} \boldsymbol{u}_{\eta}$ are bounded in $\mathbf{L}^{2}(\mathbf{T})$. Indeed, the weak formulation of (3.13) implies

$$
\int_{\mathbf{T}} \eta^{2} \varepsilon^{-1}(\boldsymbol{x}, \boldsymbol{x} / \eta)\left|\boldsymbol{c u r l} \boldsymbol{u}_{\eta}(\boldsymbol{x})\right|^{2} \mathrm{~d} \boldsymbol{x}+\int_{\mathbf{T}}\left|\boldsymbol{u}_{\eta}(\boldsymbol{x})\right|^{2} \mathrm{~d} \boldsymbol{x}=\int_{\mathbf{T}} \boldsymbol{f}_{\eta}(\boldsymbol{x}) \cdot \boldsymbol{u}_{\eta}(\boldsymbol{x}) \mathrm{d} \boldsymbol{x} .
$$

In view of the assumption that $\varepsilon(\boldsymbol{x}, \boldsymbol{y})$ is coercive and noticing that

$$
\left|\int_{\mathbf{T}} \boldsymbol{f}(\boldsymbol{x}, \boldsymbol{x} / \eta) \cdot \boldsymbol{u}_{\eta}(\boldsymbol{x}) \mathrm{d} \boldsymbol{x}\right| \leq\left\|\boldsymbol{f}_{\eta}\right\|_{\mathbf{L}^{2}(\mathbf{T})}\left\|\boldsymbol{u}_{\eta}\right\|_{\mathbf{L}^{2}(\mathbf{T})}
$$

it follows from (7.30) that

$$
\left\|\boldsymbol{u}_{\eta}\right\|_{\mathbf{L}^{2}(\mathbf{T})} \leq\left\|\boldsymbol{f}_{\eta}\right\|_{\mathbf{L}^{2}(\mathbf{T})}
$$

and

$$
\left\|\eta \operatorname{curl} \boldsymbol{u}_{\eta}\right\|_{\mathbf{L}^{2}(\mathbf{T})} \leq \nu^{-1 / 2}\left\|\boldsymbol{f}_{\eta}\right\|_{\mathbf{L}^{2}(\mathbf{T})},
$$

where $\nu>0$ is a coercivity constant for the matrix $\varepsilon^{-1}(\boldsymbol{x}, \boldsymbol{y})$. Hence up to a subsequence, $\boldsymbol{u}_{\eta} \rightarrow \boldsymbol{u}_{0}$ and $\eta \operatorname{curl} \boldsymbol{u}_{\eta} \rightarrow \boldsymbol{v}$, where $\boldsymbol{u}, \boldsymbol{v} \in \mathbf{L}^{2}(\mathbf{T} \times Y)(c f$. [1], [3], [7]). Next we establish a relation between the two-scale limits $\boldsymbol{u}_{0}$ and $\boldsymbol{v}$.

Using integration by parts, for any $\varphi \in \mathbf{C}_{\text {per }}^{\infty}(\mathbf{T} \times K Y)$ we write

$$
\eta \int_{\mathbf{T}} \operatorname{curl} \boldsymbol{u}_{\eta}(\boldsymbol{x}) \cdot \boldsymbol{\varphi}(\boldsymbol{x}, \boldsymbol{x} / \eta) \mathrm{d} \boldsymbol{x}=\left.\int_{\mathbf{T}} \eta \boldsymbol{u}_{\eta}(\boldsymbol{x}) \cdot \operatorname{curl}_{x} \boldsymbol{\varphi}(\boldsymbol{x}, \boldsymbol{y})\right|_{y=x / \eta} \mathrm{d} \boldsymbol{x}+\left.\int_{\mathbf{T}} \boldsymbol{u}_{\eta}(\boldsymbol{x}) \cdot \operatorname{curl}_{y} \boldsymbol{\varphi}(\boldsymbol{x}, \boldsymbol{y})\right|_{y=x / \eta} \mathrm{d} \boldsymbol{x} .
$$

Since $\boldsymbol{\varphi}(\boldsymbol{x}, \boldsymbol{x} / \eta)$ converges two-scale strongly to $\boldsymbol{\varphi}(\boldsymbol{x}, \boldsymbol{y})$, we can pass to the limit in (7.31) as $\eta \rightarrow 0$, which yields

$$
\int_{\mathbf{T}} \int_{K Y} \boldsymbol{v}(\boldsymbol{x}, \boldsymbol{y}) \cdot \boldsymbol{\varphi}(\boldsymbol{x}, \boldsymbol{y}) \mathrm{d} \boldsymbol{y} \mathrm{d} \boldsymbol{x}=\int_{\mathbf{T}} \int_{K Y} \boldsymbol{u}_{0}(\boldsymbol{x}, \boldsymbol{y}) \cdot \operatorname{curl}_{y} \varphi(\boldsymbol{x}, \boldsymbol{y}) \mathrm{d} \boldsymbol{y} \mathrm{d} \boldsymbol{x}
$$

Setting $\boldsymbol{\varphi}(\boldsymbol{x}, \boldsymbol{y})=\Phi(\boldsymbol{x}) \boldsymbol{\Psi}(\boldsymbol{y})$, where $\Phi \in C_{\text {per }}^{\infty}(\mathbf{T})$ and $\boldsymbol{\Psi} \in \mathbf{C}_{\text {per }}^{\infty}(K Y)$ are arbitrary, we deduce from (7.32) that for almost every $\boldsymbol{x} \in \mathbf{T}$ one has $\operatorname{curl}_{y} \boldsymbol{u}_{0}(\boldsymbol{x}, \boldsymbol{y})=\boldsymbol{v}(\boldsymbol{x}, \boldsymbol{y})$. In particular, we get $\boldsymbol{u}_{0}(\boldsymbol{x}, \boldsymbol{y}) \in$ $\mathbf{L}^{2}\left(\mathbf{T}, \mathcal{H}_{\text {per }}(\operatorname{curl}, K Y)\right)$.

Furthermore, (3.13) implies

$$
\begin{gathered}
\int_{\mathbf{T}} \varepsilon^{-1}(\boldsymbol{x}, \boldsymbol{x} / \eta) \eta \operatorname{curl} \boldsymbol{u}_{\eta}(\boldsymbol{x}) \cdot\left(\eta \operatorname{curl}_{x} \boldsymbol{\varphi}(\boldsymbol{x}, \boldsymbol{y})+\left.\operatorname{curl}_{y} \boldsymbol{\varphi}(\boldsymbol{x}, \boldsymbol{y})\right|_{y=x / \eta}\right) \mathrm{d} \boldsymbol{x} \\
+\int_{\mathbf{T}} \boldsymbol{u}_{\eta}(\boldsymbol{x}) \cdot \boldsymbol{\varphi}(\boldsymbol{x}, \boldsymbol{x} / \eta) \mathrm{d} \boldsymbol{x}=\int_{\mathbf{T}} \boldsymbol{f}_{\eta}(\boldsymbol{x}) \cdot \boldsymbol{\varphi}(\boldsymbol{x}, \boldsymbol{x} / \eta) \mathrm{d} \boldsymbol{x} .
\end{gathered}
$$

Passing to the limit in (7.33) as $\eta \rightarrow 0$, we get

$$
\begin{gathered}
\int_{\mathbf{T}} \int_{K Y} \varepsilon^{-1}(\boldsymbol{x}, \boldsymbol{y}) \operatorname{curl}_{y} \boldsymbol{u}_{0}(\boldsymbol{x}, \boldsymbol{y}) \cdot \operatorname{curl}_{y} \boldsymbol{\varphi}(\boldsymbol{x}, \boldsymbol{y}) \mathrm{d} \boldsymbol{y} \mathrm{d} \boldsymbol{x}+\int_{\mathbf{T}} \int_{K Y} \boldsymbol{u}_{0}(\boldsymbol{x}, \boldsymbol{y}) \cdot \boldsymbol{\varphi}(\boldsymbol{x}, \boldsymbol{y}) \mathrm{d} \boldsymbol{y} \mathrm{d} \boldsymbol{x} \\
=\int_{\mathbf{T}} \int_{K Y} \boldsymbol{f}(\boldsymbol{x}, \boldsymbol{y}) \cdot \boldsymbol{\varphi}(\boldsymbol{x}, \boldsymbol{y}) \mathrm{d} \boldsymbol{y} \mathrm{d} \boldsymbol{x} .
\end{gathered}
$$

Setting, again, $\boldsymbol{\varphi}(\boldsymbol{x}, \boldsymbol{y})=\Phi(\boldsymbol{x}) \boldsymbol{\Psi}(\boldsymbol{y})$, where $\Phi \in C_{\text {per }}^{\infty}(\mathbf{T})$ and $\boldsymbol{\Psi} \in \mathbf{C}_{\text {per }}^{\infty}(K Y)$, we conclude that (3.14) holds. Finally, in order to prove that $\boldsymbol{u}_{\eta} \rightarrow \boldsymbol{u}$ we show that

$$
\limsup _{\eta \rightarrow 0} \int_{\mathbf{T}}\left|\boldsymbol{u}_{\eta}(\boldsymbol{x})\right|^{2} \mathrm{~d} \boldsymbol{x} \leq \frac{1}{K^{3}} \int_{\mathbf{T}} \int_{K Y}\left|\boldsymbol{u}_{0}(\boldsymbol{x}, \boldsymbol{y})\right|^{2} \mathrm{~d} \boldsymbol{y} \mathrm{d} \boldsymbol{x} .
$$


To this end notice that the minimising properties of $\boldsymbol{u}_{\eta}$ and $\boldsymbol{u}_{0}(\boldsymbol{x}, \boldsymbol{x} / \eta)$ imply

$$
\int_{\mathbf{T}}\left|\boldsymbol{c u r l} \boldsymbol{u}_{\eta}(\boldsymbol{x})\right|^{2} \mathrm{~d} \boldsymbol{x}+\int_{\mathbf{T}}\left|\boldsymbol{u}_{\eta}(\boldsymbol{x})\right|^{2} \mathrm{~d} \boldsymbol{x}=\int_{\mathbf{T}} \boldsymbol{f}_{\eta}(\boldsymbol{x}) \cdot \boldsymbol{u}_{\eta}(\boldsymbol{x}) \mathrm{d} \boldsymbol{x}
$$

for any $\eta \in \Xi$, and

$$
\frac{1}{K^{3}} \int_{\mathbf{T}} \int_{K Y}\left|\operatorname{curl}_{y} \boldsymbol{u}_{0}(\boldsymbol{x}, \boldsymbol{y})\right|^{2} \mathrm{~d} \boldsymbol{y} \mathrm{d} \boldsymbol{x}+\frac{1}{K^{3}} \int_{\mathbf{T}} \int_{K Y}\left|\boldsymbol{u}_{0}(\boldsymbol{x}, \boldsymbol{y})\right|^{2} \mathrm{~d} \boldsymbol{y} \mathrm{d} \boldsymbol{x}=\frac{1}{K^{3}} \int_{\mathbf{T}} \int_{K Y} \boldsymbol{f}(\boldsymbol{x}, \boldsymbol{y}) \cdot \boldsymbol{u}_{0}(\boldsymbol{x}, \boldsymbol{y}) \mathrm{d} \boldsymbol{y} \mathrm{d} \boldsymbol{x},
$$

respectively. In view of the fact that the right-hand side of (7.35) converges to the right-hand side of (7.36) as $\eta \rightarrow 0$, we get

$$
\begin{aligned}
& \limsup _{\eta \rightarrow 0} \int_{\mathbf{T}}\left|\boldsymbol{u}_{\eta}(\boldsymbol{x})\right|^{2} \mathrm{~d} \boldsymbol{x} \leq \frac{1}{K^{3}} \int_{\mathbf{T}} \int_{K Y}\left|\operatorname{curl}_{y} \boldsymbol{u}_{0}(\boldsymbol{x}, \boldsymbol{y})\right|^{2} \mathrm{~d} \boldsymbol{y} \mathrm{d} \boldsymbol{x} \\
& +\frac{1}{K^{3}} \int_{\mathbf{T}} \int_{K Y}\left|\boldsymbol{u}_{0}(\boldsymbol{x}, \boldsymbol{y})\right|^{2} \mathrm{~d} \boldsymbol{y} \mathrm{d} \boldsymbol{x}-\lim \inf _{\eta \rightarrow 0} \int_{\mathbf{T}}\left|\operatorname{curl} \boldsymbol{u}_{\eta}(\boldsymbol{x})\right|^{2} \mathrm{~d} \boldsymbol{x} .
\end{aligned}
$$

The inequality

$$
\frac{1}{K^{3}} \int_{\mathbf{T}} \int_{K Y}\left|\operatorname{curl}_{y} \boldsymbol{u}_{0}(\boldsymbol{x}, \boldsymbol{y})\right|^{2} \mathrm{~d} \boldsymbol{y} \mathrm{d} \boldsymbol{x} \leq \lim \inf _{\eta \rightarrow 0} \int_{\mathbf{T}}\left|\operatorname{curl} \boldsymbol{u}_{\eta}(\boldsymbol{x})\right|^{2} \mathrm{~d} \boldsymbol{x}
$$

in conjunction with (7.37) immediately implies (7.34).

\section{Proof of Lemma 3}

Consider the sequence $\eta_{l}$ such that $\eta_{l}^{-1} 2 T=2^{2 l}$. Then the periodic cell $\mathbf{T}$ is covered by cubes $P_{i}^{l}, i=1, \ldots, 2^{3 l}$, of size $\sqrt{2 T \eta_{l}}$, whose interiors are disjoint. Denote by $\boldsymbol{x}_{i}^{l}$ the centre of the cube $P_{i}^{l}, i=1, \ldots, 2^{3 l}$, and by $i_{l}$ one of those indices $i$ for which the norm $\left\|\boldsymbol{u}_{\eta}\right\|_{\mathbf{L}^{2}\left(P_{i}^{l}\right)}$ attains its maximum over all indices $i=1, \ldots, 2^{3 l}$.

The sequence $\boldsymbol{x}_{i_{l}}^{l}$, being bounded, has a convergent subsequence, which corresponds to some indices $l_{j}$ and whose limit we denote by $\boldsymbol{x}_{0}$. In what follows we use the notation $\boldsymbol{x}_{j}$ for this subsequence, and also denote $\boldsymbol{u}_{\eta_{l_{j}}}$ by $\boldsymbol{u}_{j}, P_{i_{l_{j}}}^{l_{j}}$ by $P_{j}$, and $\eta_{l_{j}}$ by $\eta_{j}$. Also, we set $\beta_{j}:=2^{1-l_{j}} T$. Consider a positive scalar cut-off function $\varphi \in C_{0}^{\infty}\left(\mathbb{R}^{3}\right)$ such that $\operatorname{supp}(\varphi) \subset[-1,1]^{3}$ and $\varphi(\boldsymbol{x})=1$ when $\boldsymbol{x} \in[-1 / 2,1 / 2]^{3}$, and define

$$
\tilde{\boldsymbol{u}}_{j}:=\left\|\varphi_{j} \boldsymbol{u}_{j}\right\|_{\mathbf{L}^{2}(\mathbf{T})}^{-1} \varphi_{j} \boldsymbol{u}_{j}
$$

where $\varphi_{j}(\boldsymbol{x}):=\varphi\left(\beta_{j}^{-1}\left(\boldsymbol{x}-\boldsymbol{x}_{j}\right)\right)$. Further, denote $D_{j}:=\operatorname{supp}\left(\varphi_{j}\right)$. We begin by suggesting the following auxiliary statement.

Lemma 4. There is a positive constant $C$ such that for any $j$,

$$
\left\|\boldsymbol{u}_{j}\right\|_{\mathbf{L}^{2}\left(D_{j}\right)} \leq C\left\|\boldsymbol{u}_{j}\right\|_{\mathbf{L}^{2}\left(P_{j}\right)}
$$

and

$$
\eta_{j}\left\|\operatorname{curl} \boldsymbol{u}_{j}\right\|_{\mathbf{L}^{2}\left(D_{j}\right)} \leq C\left\|\boldsymbol{u}_{j}\right\|_{\mathbf{L}^{2}\left(P_{j}\right)} .
$$

Proof. The estimate (7.38) follows from the fact that $D_{j}$ is covered by at most 27 cubes from the set $\left\{P_{i}^{l_{j}}, i=1, \ldots, 2^{3 l_{j}}\right\}$ and $\left\|\boldsymbol{u}_{j}\right\|_{\mathbf{L}^{2}\left(P_{i}^{l_{j}}\right)}$ attains its maximum for $i=i_{l_{j}}$. In order to verify (7.39) consider the function $\psi_{j}(\boldsymbol{x}):=\varphi_{j}(\boldsymbol{x} / 2)$ and multiply the equation (4.15) by $\psi_{j}^{2} \boldsymbol{u}_{j}$. Integrating by parts we get

$$
\begin{gathered}
\eta_{j} \int_{\mathbf{T}} \psi_{j}^{2} \varepsilon^{-1}\left(\boldsymbol{x}, \boldsymbol{x} / \eta_{j}\right) \operatorname{curl} \boldsymbol{u}_{j} \cdot \mathbf{c u r l} \boldsymbol{u}_{j} \mathrm{~d} \boldsymbol{x} \\
=\left(\mu_{\eta_{j}}^{-1}-1\right) \int_{\mathbf{T}} \psi_{j}^{2} \boldsymbol{u}_{j} \cdot \boldsymbol{u}_{j} \mathrm{~d} \boldsymbol{x}-2 \eta_{j}^{2} \int_{\mathbf{T}} \varepsilon^{-1}\left(\boldsymbol{x}, \boldsymbol{x} / \eta_{j}\right) \psi_{j} \operatorname{curl} \boldsymbol{u}_{j} \cdot\left(\boldsymbol{u}_{j} \times \nabla \psi_{j}\right) \mathrm{d} \boldsymbol{x} .
\end{gathered}
$$

Using the fact that the matrices $\varepsilon^{-1}(\boldsymbol{x}, \boldsymbol{x} / \eta), \eta \in \Xi$, are uniformly positive-definite, we get

$$
\begin{gathered}
\eta_{j}^{2}\left\|\psi_{j} \operatorname{curl} \boldsymbol{u}_{j}\right\|_{\mathbf{L}^{2}(\mathbf{T})}^{2} \leq C\left(\eta_{j}^{2}\left\|\psi_{j} \operatorname{curl} \boldsymbol{u}_{j}\right\|_{\mathbf{L}^{2}(\mathbf{T})}\left\|\boldsymbol{u}_{j} \times \nabla \psi_{j}\right\|_{\mathbf{L}^{2}(\mathbf{T})}+\left\|\psi_{j} \boldsymbol{u}_{j}\right\|_{\mathbf{L}^{2}(\mathbf{T})}\right) \\
\leq C\left(\eta_{j}^{2} \beta_{j}^{-1}\left\|\psi_{j} \operatorname{curl} \boldsymbol{u}_{j}\right\|_{\mathbf{L}^{2}(\mathbf{T})}\left\|\boldsymbol{u}_{j}\right\|_{\mathbf{L}^{2}\left(D_{j}\right)}+\left\|\boldsymbol{u}_{j}\right\|_{\mathbf{L}^{2}\left(D_{j}\right)}^{2}\right) \\
\leq C\left(\eta_{j}\left\|\psi_{j} \operatorname{curl} \boldsymbol{u}_{j}\right\|_{\mathbf{L}^{2}(\mathbf{T})}\left\|\boldsymbol{u}_{j}\right\|_{\mathbf{L}^{2}\left(D_{j}\right)}+\left\|\boldsymbol{u}_{j}\right\|_{\mathbf{L}^{2}\left(D_{j}\right)}^{2}\right)
\end{gathered}
$$

for some constant $C>0$, from which (7.39) immediately follows, taking into account (7.38). 
Proceeding with the proof of the above proposition, notice that the sequence $\tilde{\boldsymbol{u}}_{j}$ defined above is a sequence of quasi-eigenvectors for the matrix $\varepsilon^{-1}\left(\boldsymbol{x}, \boldsymbol{x} / \eta_{j}\right)$. Indeed, for the vector-function $\tilde{\boldsymbol{r}}_{j}$ defined by

$$
\tilde{\boldsymbol{r}}_{j}:=\eta_{j}^{2} \operatorname{curl}\left(\varepsilon^{-1}\left(\boldsymbol{x}, \boldsymbol{x} / \eta_{j}\right) \operatorname{curl} \tilde{\boldsymbol{u}}_{j}\right)+\left(1-\mu_{\eta_{j}}^{-1}\right) \tilde{\boldsymbol{u}}_{j},
$$

and a sequence $\boldsymbol{w}_{j} \in \mathcal{H}_{\text {per }}(\mathbf{c u r l}, \mathbf{T})$, we have

$$
\begin{gathered}
\left\langle\tilde{\boldsymbol{r}}_{j}, \boldsymbol{w}_{j}\right\rangle_{\mathcal{H}^{-1}, \mathcal{H}_{\mathrm{per}}(\operatorname{curl}, \mathbf{T})}=\left\|\varphi_{j} \boldsymbol{u}_{j}\right\|_{\mathbf{L}^{2}(\mathbf{T})}^{-1}\left(\eta_{j}^{2} \int_{\mathbf{T}} \varepsilon^{-1}\left(\boldsymbol{x}, \boldsymbol{x} / \eta_{j}\right) \operatorname{curl}\left(\varphi_{j} \boldsymbol{u}_{j}\right) \cdot \operatorname{curl} \boldsymbol{w}_{j} \mathrm{~d} \boldsymbol{x}\right. \\
\left.-\eta_{j}^{2} \int_{\mathbf{T}} \varepsilon^{-1}\left(\boldsymbol{x}, \boldsymbol{x} / \eta_{j}\right) \operatorname{curl} \boldsymbol{u}_{j} \cdot \operatorname{curl}\left(\varphi_{j} \boldsymbol{w}_{j}\right)\right) \mathrm{d} \boldsymbol{x} \\
=\left\|\varphi_{j} \boldsymbol{u}_{j}\right\|_{\mathbf{L}^{2}(\mathbf{T})}^{-1} \eta_{j}^{2} \int_{\mathbf{T}} \varepsilon^{-1}\left(\boldsymbol{x}, \boldsymbol{x} / \eta_{j}\right) \nabla \varphi_{j} \cdot\left(\boldsymbol{u}_{j} \times \operatorname{curl} \boldsymbol{w}_{j}-\boldsymbol{w}_{j} \times \operatorname{curl} \boldsymbol{u}_{j}\right) \mathrm{d} \boldsymbol{x}
\end{gathered}
$$

Therefore, we have

$$
\left|\left\langle\tilde{\boldsymbol{r}}_{j}, \boldsymbol{w}_{j}\right\rangle_{\mathcal{H}^{-1}, \mathcal{H}_{\mathrm{per}}(\mathbf{c u r l}, \mathbf{T})}\right| \leq\left\|\boldsymbol{u}_{j}\right\|_{\mathbf{L}^{2}\left(P_{j}\right)}^{-1} \eta_{j}^{2} \beta_{j}^{-1}\left(\left\|\boldsymbol{u}_{j}\right\|_{\mathbf{L}^{2}\left(D_{j}\right)}\left\|\operatorname{curl} \boldsymbol{w}_{j}\right\|_{\mathbf{L}^{2}(\mathbf{T})}+\left\|\boldsymbol{w}_{j}\right\|_{\mathbf{L}^{2}(\mathbf{T})}\left\|\operatorname{curl} \boldsymbol{u}_{j}\right\|_{\mathbf{L}^{2}\left(D_{j}\right)}\right) .
$$

In view of the above lemma, the last estimate implies

$$
\left|\left\langle\tilde{\boldsymbol{r}}_{j}, \boldsymbol{w}_{j}\right\rangle_{\mathcal{H}^{-1}, \mathcal{H}_{\mathrm{per}}(\mathbf{c u r l}, \mathbf{T})}\right| \leq C \eta_{j} \beta_{j}^{-1}\left(\left\|\boldsymbol{w}_{j}\right\|_{\mathbf{L}^{2}(\mathbf{T})}+\eta_{j}\left\|\operatorname{curl} \boldsymbol{w}_{j}\right\|_{\mathbf{L}^{2}(\mathbf{T})}\right),, m
$$

hence (4.16) with $\boldsymbol{r}_{j}=\tilde{\boldsymbol{r}}_{j}$. Finally, to prove that $\tilde{\boldsymbol{u}}_{j}$ is a sequence of quasi-eigenfunctions for the matrix $\varepsilon^{-1}\left(\boldsymbol{x}_{0}, \boldsymbol{x} / \eta_{j}\right)$, note that for $\boldsymbol{r}_{j}$ defined by

$$
\boldsymbol{r}_{j}:=\eta_{j}^{2} \operatorname{curl}\left(\varepsilon^{-1}\left(\boldsymbol{x}_{0}, \boldsymbol{x} / \eta_{j}\right) \operatorname{curl} \tilde{\boldsymbol{u}}_{j}\right)+\left(1-\mu_{j}^{-1}\right) \tilde{\boldsymbol{u}}_{j}
$$

and a sequence $\boldsymbol{w}_{j} \in \mathcal{H}_{\text {per }}(\mathbf{T})$ such that the norms $\left\|\boldsymbol{w}_{j}\right\|_{\mathbf{L}^{2}(\mathbf{T})}+\eta_{j}\left\|\mathbf{c u r l} \boldsymbol{w}_{j}\right\|_{\mathbf{L}^{2}(\mathbf{T})}$ are uniformly bounded, one has

$$
\begin{gathered}
\left\langle\boldsymbol{r}_{j}, \boldsymbol{w}_{j}\right\rangle_{\mathcal{H}^{-1}, \mathcal{H}_{\mathrm{per}}(\mathbf{c u r l}, \mathbf{T})}=\left\langle\tilde{\boldsymbol{r}}_{j}, \boldsymbol{w}_{j}\right\rangle_{\mathcal{H}^{-1}, \mathcal{H}_{\mathrm{per}}(\mathbf{c u r l}, \mathbf{T})} \\
+\eta_{j}^{2} \int_{\mathbf{T}}\left(\varepsilon^{-1}\left(\boldsymbol{x}_{0}, \boldsymbol{x} / \eta_{j}\right)-\varepsilon^{-1}\left(\boldsymbol{x}, \boldsymbol{x} / \eta_{j}\right)\right) \operatorname{curl} \tilde{\boldsymbol{u}}_{j} \cdot \operatorname{curl} \boldsymbol{w}_{j} \mathrm{~d} \boldsymbol{x} .
\end{gathered}
$$

The first term in the right-hand side of (7.41) vanishes as $j \rightarrow \infty$ in view of the fact that $\tilde{\boldsymbol{u}}_{j}$ is a sequence of quasi-eigenfunctions for the matrix $\varepsilon^{-1}\left(\boldsymbol{x}, \boldsymbol{x} / \eta_{j}\right)$, while the second term is bounded by

$$
\eta_{j}^{2}\left\|\operatorname{curl} \boldsymbol{w}_{j}\right\|_{\mathbf{L}^{2}(\mathbf{T})}\left\|\operatorname{curl} \tilde{\boldsymbol{u}}_{j}\right\|_{\mathbf{L}^{2}(\mathbf{T})} \sup _{x \in D_{j}}\left\|\varepsilon^{-1}\left(\boldsymbol{x}_{0}, \cdot\right)-\varepsilon^{-1}(\boldsymbol{x}, \cdot)\right\|_{\left[L^{\infty}(Y)\right]^{9}},
$$

which also vanishes as $j \rightarrow \infty$, since $D_{j}=\operatorname{supp}\left(\varphi_{j}\right)$ concentrates near the point $\boldsymbol{x}_{0}$.

\section{Proof of Theorem 3}

i) The existence and uniqueness of a solution $\boldsymbol{u}_{\eta} \in \mathcal{H}_{\text {per }}(\mathbf{c u r l}, \mathbf{T})$ to (5.22) follows from the Lax-Milgram lemma (see e.g. [10]).

ii) The sequences $\boldsymbol{u}_{\eta}$ and $a_{\eta} \operatorname{curl} \boldsymbol{u}_{\eta}$ are bounded in $\mathbf{L}^{2}(\mathbf{T})$. The proof is of this fact is analogous to that given in Theorem 1. Hence, for a fixed $l \in \mathbb{N}$ and up to a subsequence ${ }^{9}, \boldsymbol{u}_{\eta} \boldsymbol{w} \boldsymbol{v}_{0}$ and $a_{\eta} \operatorname{curl} \boldsymbol{u}_{\eta} \boldsymbol{-} \boldsymbol{v}_{1}$, where $\boldsymbol{v}_{0}, \boldsymbol{v}_{1} \in \mathbf{L}^{2}(\mathbf{T} \times Z \times Y)$. We start by studying in more detail the structure of $\boldsymbol{v}_{0}$ and the relationship between $\boldsymbol{v}_{0}$ and $\boldsymbol{v}_{1}$.

To this end, notice first that for any $\varphi \in \mathbf{C}_{\mathrm{per}}^{\infty}(\mathbf{T} \times Z \times Y)$,

$$
\begin{aligned}
& \int_{\mathbf{T}} \operatorname{curl} \boldsymbol{u}_{\eta}(\boldsymbol{x}) \cdot a_{\eta} \boldsymbol{\varphi}\left(\boldsymbol{x}, \boldsymbol{x} / a_{\eta}, \boldsymbol{x} / \eta\right) \mathrm{d} \boldsymbol{x}=\left.\int_{\mathbf{T}} \boldsymbol{u}_{\eta}(\boldsymbol{x}) \cdot a_{\eta} \operatorname{curl}_{x} \boldsymbol{\varphi}(\boldsymbol{x}, \boldsymbol{z}, \boldsymbol{y})\right|_{z=x / a_{\eta}, y=x / \eta} \mathrm{d} \boldsymbol{x} \\
&+\left.\int_{\mathbf{T}} \boldsymbol{u}_{\eta}(\boldsymbol{x}) \cdot \operatorname{curl}_{z} \boldsymbol{\varphi}(\boldsymbol{x}, \boldsymbol{z}, \boldsymbol{y})\right|_{z=x / a_{\eta}, y=x / \eta} \mathrm{d} \boldsymbol{x} .
\end{aligned}
$$

Passing to the limit in this identity yields

$$
\int_{\mathbf{T}} \int_{Z} \int_{Y} \boldsymbol{v}_{0}(\boldsymbol{x}, \boldsymbol{z}, \boldsymbol{y}) \cdot \operatorname{curl}_{y} \boldsymbol{\varphi}(\boldsymbol{x}, \boldsymbol{z}, \boldsymbol{y}) \mathrm{d} \boldsymbol{y} \mathrm{d} \boldsymbol{z} \mathrm{d} \boldsymbol{x}=0,
$$

\footnotetext{
${ }^{9}$ Here we make use of the fact that three-scale convergence possesses a compactness property analogous to that for two-scale convergence, $c f$. the text after Definition 1.
} 
which implies $\boldsymbol{v}_{0}=\boldsymbol{u}_{0}+\boldsymbol{u}_{1}$, where $\boldsymbol{u}_{0}$ is the $\boldsymbol{y}$-gradient of a function from $L^{2}\left(\mathbf{T} \times Z, H_{\mathrm{per}}^{1}(Y)\right)$, and $\boldsymbol{u}_{1}$ in independent of $\boldsymbol{y}$. Next, for any $\boldsymbol{\varphi} \in \mathbf{C}_{\mathrm{per}}^{\infty}(\mathbf{T} \times Z \times Y)$, we have

$$
\begin{gathered}
\int_{\mathbf{T}} a_{\eta} \operatorname{curl}_{\eta}(\boldsymbol{x}) \cdot \boldsymbol{\varphi}\left(\boldsymbol{x}, \boldsymbol{x} / a_{\eta}, \boldsymbol{x} / \eta\right) \mathrm{d} \boldsymbol{x}=\left.\int_{\mathbf{T}} a_{\eta} \boldsymbol{u}_{\eta}(\boldsymbol{x}) \cdot \operatorname{curl}_{x} \boldsymbol{\varphi}(\boldsymbol{x}, \boldsymbol{z}, \boldsymbol{y})\right|_{z=x / a_{\eta}, y=x / \eta} \mathrm{d} \boldsymbol{x} \\
\left.\int_{\mathbf{T}} \boldsymbol{u}_{\eta}(\boldsymbol{x}) \cdot \operatorname{curl}_{z} \boldsymbol{\varphi}(\boldsymbol{x}, \boldsymbol{z}, \boldsymbol{y})\right|_{z=x / a_{\eta}, y=x / \eta} \mathrm{d} \boldsymbol{x}+\left.\int_{\mathbf{T}} \eta^{-1} a_{\eta} \boldsymbol{u}_{\eta}(\boldsymbol{x}) \cdot \operatorname{curl}_{y} \boldsymbol{\varphi}(\boldsymbol{x}, \boldsymbol{z}, \boldsymbol{y})\right|_{z=x / a_{\eta}, y=x / \eta} \mathrm{d} \boldsymbol{x} .
\end{gathered}
$$

Passing to the limit in the last identity yields

$$
\begin{gathered}
\int_{\mathbf{T}} \int_{Z} \int_{Y} \boldsymbol{v}_{1}(\boldsymbol{x}, \boldsymbol{z}, \boldsymbol{y}) \cdot \boldsymbol{\varphi}(\boldsymbol{x}, \boldsymbol{z}, \boldsymbol{y}) \mathrm{d} \boldsymbol{y} \mathrm{d} \boldsymbol{z} \mathrm{d} \boldsymbol{x}= \\
\int_{\mathbf{T}} \int_{Z} \int_{Y} \boldsymbol{v}_{0}(\boldsymbol{x}, \boldsymbol{z}, \boldsymbol{y}) \cdot \boldsymbol{c u r l}_{z} \varphi(\boldsymbol{x}, \boldsymbol{z}, \boldsymbol{y}) \mathrm{d} \boldsymbol{y} \mathrm{d} \boldsymbol{z} \mathrm{d} \boldsymbol{x}+\left.\lim _{\eta \rightarrow 0} \int_{\mathbf{T}} \eta^{-1} a_{\eta} \boldsymbol{u}_{\eta}(\boldsymbol{x}) \cdot \operatorname{curl}_{y} \varphi(\boldsymbol{x}, \boldsymbol{z}, \boldsymbol{y})\right|_{z=x / a_{\eta}, y=x / \eta} \mathrm{d} \boldsymbol{x} .
\end{gathered}
$$

Setting in (7.42) $\varphi=\varphi(\boldsymbol{x}, \boldsymbol{z})$, we get

$$
\int_{\mathbf{T}} \int_{Z}\left\langle\boldsymbol{v}_{1}(\boldsymbol{x}, \boldsymbol{z}, \boldsymbol{y})\right\rangle_{y} \cdot \boldsymbol{\varphi}(\boldsymbol{x}, \boldsymbol{z}) \mathrm{d} \boldsymbol{z} \mathrm{d} \boldsymbol{x}=\int_{\mathbf{T}} \int_{Z}\left\langle\boldsymbol{v}_{0}(\boldsymbol{x}, \boldsymbol{z}, \boldsymbol{y})\right\rangle_{y} \cdot \operatorname{curl}_{z} \boldsymbol{\varphi}(\boldsymbol{x}, \boldsymbol{z}) \mathrm{d} \boldsymbol{z} \mathrm{d} \boldsymbol{x} .
$$

Note that $\left\langle\boldsymbol{v}_{0}(\boldsymbol{x}, \boldsymbol{z}, \boldsymbol{y})\right\rangle_{y}=\boldsymbol{u}_{1}(\boldsymbol{x}, \boldsymbol{z})$, therefore $\boldsymbol{u}_{1} \in L^{2}\left(\mathbf{T}, \mathcal{H}_{\mathrm{per}}(\operatorname{curl}, Z)\right)$ and $\operatorname{curl}_{z} \boldsymbol{u}_{1}(\boldsymbol{x}, \boldsymbol{z})=\left\langle\boldsymbol{v}_{1}(\boldsymbol{x}, \boldsymbol{z}, \boldsymbol{y})\right\rangle_{y}$. The identity (7.42) can now be rewritten as

$$
\begin{gathered}
\int_{\mathbf{T}} \int_{Z} \int_{Y}\left(\boldsymbol{v}_{1}(\boldsymbol{x}, \boldsymbol{z}, \boldsymbol{y})-\operatorname{curl}_{z} \boldsymbol{u}_{1}(\boldsymbol{x}, \boldsymbol{z})\right) \cdot \boldsymbol{\varphi}(\boldsymbol{x}, \boldsymbol{z}, \boldsymbol{y}) \mathrm{d} \boldsymbol{y} \mathrm{d} \boldsymbol{z} \mathrm{d} \boldsymbol{x}= \\
\int_{\mathbf{T}} \int_{Z} \int_{Y} \boldsymbol{u}_{0}(\boldsymbol{x}, \boldsymbol{z}, \boldsymbol{y}) \cdot \operatorname{curl}_{z} \boldsymbol{\varphi}(\boldsymbol{x}, \boldsymbol{z}, \boldsymbol{y}) \mathrm{d} \boldsymbol{y} \mathrm{d} \boldsymbol{z} \mathrm{d} \boldsymbol{x}+\left.\lim _{\eta \rightarrow 0} \int_{\mathbf{T}} \eta^{-1} a_{\eta} \boldsymbol{u}_{\eta}(\boldsymbol{x}) \cdot \operatorname{curl}_{y} \boldsymbol{\varphi}(\boldsymbol{x}, \boldsymbol{z}, \boldsymbol{y})\right|_{z=x / a_{\eta}, y=x / \eta} \mathrm{d} \boldsymbol{x} .
\end{gathered}
$$

Finally, setting in (7.43) $\boldsymbol{\varphi}(\boldsymbol{x}, \boldsymbol{z}, \boldsymbol{y})=\nabla_{y} \psi(\boldsymbol{x}, \boldsymbol{z}, \boldsymbol{y})$, where $\psi \in C_{\mathrm{per}}^{\infty}(\mathbf{T} \times Z \times Y)$, and using the identity $\operatorname{curl}_{z} \nabla_{y} \psi=-\operatorname{curl}_{y} \nabla_{z} \psi$, yields

$\int_{\mathrm{T}} \int_{Z} \int_{Y}\left(\boldsymbol{v}_{1}(\boldsymbol{x}, \boldsymbol{z}, \boldsymbol{y})-\operatorname{curl}_{z} u_{1}(\boldsymbol{x}, \boldsymbol{z})\right) \cdot \nabla \psi(\boldsymbol{x}, \boldsymbol{z}, \boldsymbol{y}) \mathrm{d} \boldsymbol{y} \mathrm{d} \boldsymbol{z} \mathrm{d} \boldsymbol{x}=\int_{\mathrm{T}} \int_{Z} \int_{Y} \boldsymbol{u}_{0}(\boldsymbol{x}, \boldsymbol{z}, \boldsymbol{y}) \cdot \operatorname{curl}_{y} \nabla_{z} \psi(\boldsymbol{x}, \boldsymbol{z}, \boldsymbol{y}) \mathrm{d} \boldsymbol{y} \mathrm{d} \boldsymbol{z} \mathrm{d} \boldsymbol{x}$, where the last term vanishes in view of the fact that $\boldsymbol{u}_{0}$ is a potential vector in the variable $\boldsymbol{y}$. Hence, $\boldsymbol{v}_{1}-\operatorname{curl}_{z} \boldsymbol{u}_{1} \in L^{2}\left(\mathbf{T} \times Z, L_{\mathrm{sol}}^{2}(Y)\right)$.

We next use the weak formulation of (5.22), and for any $\varphi_{0} \in \mathbf{C}_{\text {per }}^{\infty}(\mathbf{T} \times Z), \varphi_{1} \in \mathbf{C}_{\text {per }}^{\infty}(\mathbf{T} \times Z \times Y)$ write

$$
\begin{gathered}
\left.\int_{\mathbf{T}} \varepsilon^{-1}(\boldsymbol{x}, \boldsymbol{x} / \eta) a_{\eta} \operatorname{curl} \boldsymbol{u}_{\eta}(\boldsymbol{x}) \cdot\left(a_{\eta} \operatorname{curl}_{x} \boldsymbol{\varphi}_{0}(\boldsymbol{x}, \boldsymbol{z})+\operatorname{curl}_{z} \boldsymbol{\varphi}_{0}(\boldsymbol{x}, \boldsymbol{z})\right)\right|_{\left.z=x / a_{\eta}\right)} \mathrm{d} \boldsymbol{x} \\
+\left.\int_{\mathbf{T}} \varepsilon^{-1}(\boldsymbol{x}, \boldsymbol{x} / \eta) a_{\eta} \boldsymbol{\operatorname { c u r l }} \boldsymbol{u}_{\eta}(\boldsymbol{x}) \cdot\left(\eta \operatorname{curl}_{x} \boldsymbol{\varphi}_{1}(\boldsymbol{x}, \boldsymbol{z}, \boldsymbol{y})+a_{\eta}^{-1} \eta \operatorname{curl}_{z} \boldsymbol{\varphi}_{1}(\boldsymbol{x}, \boldsymbol{z}, \boldsymbol{y})+\operatorname{curl}_{y} \boldsymbol{\varphi}_{1}(\boldsymbol{x}, \boldsymbol{y}, \boldsymbol{z})\right)\right|_{z=x / \alpha_{\eta}, y=x / \eta} \mathrm{d} \boldsymbol{x} \\
+\int_{\mathbf{T}} \boldsymbol{u}_{\eta}(\boldsymbol{x}) \cdot\left(\boldsymbol{\varphi}_{0}\left(\boldsymbol{x}, \boldsymbol{x} / a_{\eta}\right)+a_{\eta}^{-1} \eta \boldsymbol{\varphi}_{1}\left(\boldsymbol{x}, \boldsymbol{x} / a_{\eta}, \boldsymbol{x} / \eta\right)\right) \mathrm{d} \boldsymbol{x}=\int_{\mathbf{T}} \boldsymbol{f}_{\eta}(\boldsymbol{x}) \cdot\left(\boldsymbol{\varphi}_{0}\left(\boldsymbol{x}, \boldsymbol{x} / a_{\eta}\right)+a_{\eta}^{-1} \eta \boldsymbol{\varphi}_{1}\left(\boldsymbol{x}, \boldsymbol{x} / a_{\eta}, \boldsymbol{x} / \eta\right)\right) \mathrm{d} \boldsymbol{x} .
\end{gathered}
$$

Passing to the limit as $\eta \rightarrow 0$ yields

$$
\begin{gathered}
\int_{\mathbf{T}} \int_{Z} \int_{Y} \varepsilon^{-1}(\boldsymbol{x}, \boldsymbol{y}) \boldsymbol{v}_{0}(\boldsymbol{x}, \boldsymbol{y}, \boldsymbol{z}) \cdot\left(\operatorname{curl}_{z} \boldsymbol{\varphi}_{0}(\boldsymbol{x}, \boldsymbol{z})+\operatorname{curl}_{y} \boldsymbol{\varphi}_{1}(\boldsymbol{x}, \boldsymbol{z}, \boldsymbol{y})\right) \mathrm{d} \boldsymbol{y} \mathrm{d} \boldsymbol{z} \mathrm{d} \boldsymbol{x} \\
+\int_{\mathbf{T}} \int_{Z} \boldsymbol{u}_{0}(\boldsymbol{x}, \boldsymbol{z}) \cdot \boldsymbol{\varphi}_{0}(\boldsymbol{x}, \boldsymbol{z}) \mathrm{d} \boldsymbol{z} \mathrm{d} \boldsymbol{x}=\int_{\mathbf{T}} \int_{Z} \boldsymbol{f}(\boldsymbol{x}, \boldsymbol{z}) \cdot \boldsymbol{\varphi}_{0}(\boldsymbol{x}, \boldsymbol{z}) \mathrm{d} \boldsymbol{z} \mathrm{d} \boldsymbol{x} .
\end{gathered}
$$

Via a standard argument, we deduce from (7.44) that $\boldsymbol{u}_{1}$ satisfies the equation (5.23) for almost every $\boldsymbol{x} \in \mathbf{T}$.

Notice that $\boldsymbol{u}_{\eta} \rightarrow \boldsymbol{u}_{0}+\boldsymbol{u}_{1}$ clearly implies $\boldsymbol{u}_{\eta} \rightarrow \boldsymbol{u}_{1}$, where the two-scale convergence is taken with respect to the variables $\boldsymbol{x}$ and $\boldsymbol{z}$, and similarly $a_{\eta} \operatorname{curl} \boldsymbol{u}_{\eta} \rightarrow \operatorname{curl}_{z} \boldsymbol{u}_{1}$. Arguing as in the proof of Theorem 1, we prove that

$$
\lim \sup _{\eta \rightarrow 0} \int_{\mathbf{T}}\left|\boldsymbol{u}_{\eta}(\boldsymbol{x})\right|^{2} \mathrm{~d} \boldsymbol{x} \leq \int_{\mathbf{T}} \int_{Z}\left|\boldsymbol{u}_{1}(\boldsymbol{x}, \boldsymbol{z})\right|^{2} \mathrm{~d} \boldsymbol{z} \mathrm{d} \boldsymbol{x},
$$

hence $\boldsymbol{u}_{\eta} \rightarrow \boldsymbol{u}_{1}$. The statement concerning the strong two-scale convergence of the operators $\mathcal{S}_{\eta}$ follows. 


\section{References}

[1] Allaire, G. 1992 Homogenization and two-scale convergence, SIAM J. Math. Anal. 23, 1482-1518

[2] Allaire, G., Conca C. 1996 Bloch wave homogenization for a spectral problem in fluid-solid structures, Arch. Rational Mech. Anal. 35, No. 3, 197-257

[3] Allaire, G., Conca C. 1998 Bloch wave homogenization and spectral asymptotic analysis, J. Math. Pures. Appl. 77, 153-208

[4] Allaire, G., Conca C. 1998 Boundary layers in the homogenization of a spectral problem in fluid-solid structures, SIAM J. Math. Anal. 29, 343-379

[5] Allaire, G., Briane, M. 1996 Multiscale convergence and reiterated homogenization, Proc. Roy. Soc. Edinburgh 126 A, 297-342

[6] Cherednichenko, K. D., Smyshlyaev V. P., Zhikov, V. V. 2006 Non-local homogenised limits for composite media with highly anisotropic periodic fibres, Proc. Roy. Soc. Edinburgh 136 A, 87-114

[7] Conca, C., Planchard, J., Vanninathan, M. 1995 Fluids and periodic structures, RMA 38, J. Wiley and Masson, Paris

[8] Guenneau, S. 2001 Homogénéisation des quasi-cristaux et analyse des modes dans des fibres optiques de type cristal photonique, $\mathrm{PhD}$ Thesis, Université de Provence

[9] Guenneau, S., Zolla, F. 2000 Homogenization of three-dimensional finite photonic crystals, J. Elec. Waves and Appl. 14, 529-530

[10] Jikov, V. V., Kozlov, S. M., Oleinik, O. A. 1994 Homogenization of differential operators and integral functionals, Springer

[11] Lorenz. L. V. 1869 Experimentale og theoretiske undersøgelser over legemernes brydningsforhold, I, K. Dan. Vidensk. Selsk. Forh. 8(5) 203-248

[12] Nguetseng, G. 1989 A general convergence result for a functional related to the theory of homogenisation, SIAM J. Math. Anal. 20, 608-623

[13] Poulton, C. G., Botten, L. C., McPhedran, R. C., Nicorovici, N. A., Movchan, A. B. 2001 Noncommuting limits in electromagnetic scattering: asymptotic analysis for an array of highly conducting inclusions, SIAM J. Appl. Math. 61(5), 1706-1730

[14] Reed, M., Simon, B. 1978 Methods of Modern Mathematical Physics IV: Analysis of Operators, Academic Press

[15] Riesz, F., Sz.-Nagy, B. 1990 Functional Analysis, Dover

[16] Yablonovitch, E. 2001 Photonic crystals: semiconductors of light, Sci. Amer. 285, 34-41

[17] Zhikov, V. V. 2000 On an extension of the method of two-scale convergence and its applications, $S b$. Math., 191(7), 973-1014

[18] Zolla, F., Guenneau, S. 2003 A duality relation for the Maxwell system, Phys. Rev. E 67, 026610.1026610.8 